\title{
The Effective Longitudinal Dielectric Constant for Plasmas in Inhomogeneous Magnetic Fields
}

\author{
R. Gaelzer ${ }^{1}$, L. F. Ziebell ${ }^{2}$, and R. S. Schneider ${ }^{2}$ \\ ${ }^{1}$ Instituto de Física e Matemática, UFPel, Caixa Postal 354, 96010-900 Pelotas, RS, Brazil, \\ ${ }^{2}$ Instituto de Física, UFRGS, Caixa Postal 15051, 91501-970 Porto Alegre, RS, Brazil
}

Received on 26 January, 2004

\begin{abstract}
We present a detailed derivation of the effective dielectric constant to be used in the dispersion relation for electrostatic waves in the case of a plasma immersed in a inhomogeneous magnetic field, with inhomogeneity perpendicular to the direction of the magnetic field.
\end{abstract}

\section{Introduction}

We have recently discussed the correct form of the dispersion relation for electrostatic waves in inhomogeneous plasmas, considering for simplicity the particular case in which the magnetic field is homogeneous and other plasma parameters can be inhomogeneous, and deriving a general expression for the dielectric constant, valid for arbitrary direction of propagation [1-3] The situation in which the magnetic field is homogeneous has been chosen for these studies because it features a relatively simple geometry, which has been useful for the discussion of basic features, like the symmetry properties of the effective dielectric tensor. More general situations which also feature inhomogeneity in the magnetic field introduce considerable difficulty to the derivation of the effective dielectric tensor and to the effective dielectric constant, since the inhomogeneity in the magnetic field affects the resonance condition [4,5].

From the derivation presented in Refs. [3] and [2], it is important to remember here that the dispersion relation for electrostatic waves in inhomogeneous plasmas can be written in a general form which is well known from the literature [6],

$$
k^{2} \varepsilon_{l}-i \mathbf{k} \cdot(\nabla \cdot \overleftrightarrow{\varepsilon})=0
$$

where the $\overleftrightarrow{\varepsilon}$ is the dielectric tensor and $\varepsilon_{l}$ is the longitudinal dielectric constant,

$$
\varepsilon_{l}=\sum_{i j} \frac{k_{i} \varepsilon_{i j} k_{j}}{k^{2}} .
$$

It is also important to remember here that it has been demonstrated that for an inhomogeneous plasma the dielectric properties are given by the so-called effective dielectric tensor, instead of the conventional dielectric tensor $[2,7,8]$. When studying electrostatic waves, therefore, Eq. (1) must be utilized along with an effective dielectric tensor and an effective dielectric constant, derived according to Eq. (2), with the components of the effective dielectric tensor appearing in the right-hand side.
In the present paper we derive the effective dielectric constant considering the case of a magnetic field featuring perpendicular gradients, therefore complementing the formulation appearing in Refs. [2,3]. The geometry to be used is the same geometry which has been adopted in Ref. [5], which has been concerned with the general dispersion relation for electromagnetic waves. The derivation requires a considerable amount of algebraic work whose main steps are illustrated in the following sections. Due to the details which must be provided, we emphasize analytical features of the derivation, leaving detailed applications with numerical results to forthcoming publications.

The structure of the paper is the following: In section 2 the formulation employed in Ref. [5] is adopted and applied to the derivation of a general expression for the effective dielectric constant, and in section III the general expression is particularized to the case in which the plasma particles possess a Maxwellian distribution function.

\section{Derivation of the longitudinal di- electric constant}

Let us assume a plasma immersed into an inhomogeneous magnetic field, with weak inhomogeneity perpendicular to the direction of $\mathbf{B}$. Using Eq. (3) from Ref. [5]:

$$
\begin{aligned}
\overleftrightarrow{\varepsilon}=\overleftrightarrow{1}- & i \sum_{\alpha} \frac{4 \pi q_{\alpha}^{2}}{m_{\alpha} \omega} \sum_{n \rightarrow-\infty}^{\infty} \int_{0}^{\infty} d \tau \int d^{3} u u_{\perp} \mathcal{L}\left(f_{\alpha 0}\right) \\
& \times \mathrm{e}^{i D_{n \alpha} \tau}\left[F_{n \alpha}(\tau)\right]^{(|n|-1)} \frac{\Pi_{n \alpha}^{-} \Pi_{n \alpha}^{+}}{\left(x_{n}^{-} x_{n}^{+}\right)^{|n|}} \\
& -\mathbf{e}_{z} \mathbf{e}_{z} \sum_{\alpha} \frac{4 \pi q_{\alpha}^{2}}{m_{\alpha} \omega^{2}} \int d^{3} u \frac{u_{\|}}{\gamma} L\left(f_{\alpha 0}\right),
\end{aligned}
$$

where 


$$
\begin{gathered}
\Pi_{n \alpha}^{ \pm}= \pm\left[n J_{|n|}\left(x_{n}^{ \pm}\right) G_{n \alpha}^{ \pm}(\tau)+i \frac{J_{|n|+1}\left(x_{n}^{ \pm}\right)}{x_{n}^{ \pm}} F_{n \alpha}^{1 / 2}(\tau) b_{\alpha} \sin \psi\right] \mathbf{e}_{x} \\
+i\left[|n| J_{|n|}\left(x_{n}^{ \pm}\right) G_{n \alpha}^{ \pm}(\tau) \mp \frac{J_{|n|+1}\left(x_{n}^{ \pm}\right)}{x_{n}^{ \pm}} F_{n \alpha}^{1 / 2}(\tau)\left(b_{\alpha} \cos \psi \pm \mathcal{K}_{n} \tau\right)\right] \mathbf{e}_{y} \\
+\frac{u_{\|}}{u_{\perp}} J_{|n|}\left(x_{n}^{ \pm}\right) F_{n \alpha}^{1 / 2}(\tau) \mathbf{e}_{z}
\end{gathered}
$$

$$
\begin{gathered}
G_{n \alpha}^{ \pm}(\tau)=i \sqrt{\frac{\mathcal{K}_{n} \tau \mp b_{\alpha} \cos \psi+i S_{n} b_{\alpha} \sin \psi}{\mathcal{K}_{n} \tau \pm b_{\alpha} \cos \psi+i S_{n} b_{\alpha} \sin \psi}} \\
G_{n \alpha}^{+} G_{n \alpha}^{-}=-1 \\
F_{n \alpha}^{1 / 2} G_{n \alpha}^{ \pm}=-\left(\mathcal{K}_{n} \tau \mp b_{\alpha} \cos \psi+i S_{n} b_{\alpha} \sin \psi\right) .
\end{gathered}
$$

with

$$
F_{n \alpha}(\tau)=b_{\alpha}^{2}-\mathcal{K}_{n}^{2} \tau^{2}-i 2 S_{n} b_{\alpha} \sin \psi \mathcal{K}_{n} \tau
$$

In these expressions we have

$$
\begin{gathered}
D_{n \alpha}=\gamma \omega-c k_{\|} u_{\|}-n \Omega_{\alpha}\left(1+\epsilon_{B} x\right)-\epsilon_{B} \frac{k_{\perp} u_{\perp}^{2} c^{2}}{2 \Omega_{\alpha}} \sin \psi \\
b_{\alpha}=\frac{k_{\perp} u_{\perp} c}{\Omega_{\alpha}} \\
\mathcal{L}\left(f_{\alpha 0}\right)=\partial_{u_{\perp}} f_{\alpha 0}-\frac{N_{\|} u_{\perp}}{\gamma} L\left(f_{\alpha 0}\right) \\
L\left(f_{\alpha 0}\right)=\frac{u_{\|}}{u_{\perp}} \partial_{u_{\perp}} f_{\alpha 0}-\partial_{u_{\|}} f_{\alpha 0}
\end{gathered}
$$

The geometry utilized has been the following. The magnetic field has been considered pointing in the $z$ direction, and inhomogeneous in the $x$ direction, $\mathbf{B}_{0}=B_{0}(x) \mathbf{e}_{z}$. The waves were assumed propagating in arbitrary directions, with $k_{\|}$and $k_{\perp}$ as the components of the wave vector respectively parallel and perpendicular to the magnetic field. The wave angular frequency has been denoted as $\omega . \psi$ denotes the angle between the vector $\mathbf{k}_{\perp}$ and the direction of the inhomogeneity. The inhomogeneity has been assumed to be weak, such that the cyclotron frequency has been written as

$$
\Omega_{\alpha}\left(x_{\alpha}^{\prime}\right) \sim \Omega_{\alpha}(x)\left[1+\epsilon_{B}\left(x_{\alpha}^{\prime}-x\right)\right],
$$

where $\Omega_{\alpha}(x)=\left(q_{\alpha} B_{0}(x) / m_{\alpha} c\right)$ is the particle cyclotron angular frequency, $m_{\alpha}$ is the particle rest mass, $q_{\alpha}$ is the particle charge, $c$ is the velocity of light, $\epsilon_{B} \equiv\left[\frac{1}{B_{0}} \frac{d B_{0}}{d x_{\alpha}^{\prime}}\right]_{x_{\alpha}^{\prime}=x}$, and $x_{\alpha}^{\prime}$ is the unperturbed position of particle $\alpha$.

We also used

$$
\begin{gathered}
\mathbf{u}=\mathbf{p} /\left(m_{\alpha} c\right) S_{n}=\operatorname{sign}(n), \mathcal{K}_{n}=n \epsilon_{B} c u_{\perp} / 2, \\
x_{n}^{ \pm}=\left[b_{\alpha}^{2} \pm 2 b_{\alpha} \cos \psi \mathcal{K}_{n} \tau+\mathcal{K}_{n}^{2} \tau^{2}\right]^{1 / 2}
\end{gathered}
$$

The longitudinal dielectric constant can be obtained from the effective dielectric tensor as follows,

$$
\begin{aligned}
\varepsilon_{l}=1 & -i \sum_{\alpha} \frac{4 \pi q_{\alpha}^{2}}{m_{\alpha} \omega k^{2}} \sum_{n \rightarrow-\infty}^{\infty} \int_{0}^{\infty} d \tau \int d^{3} u u_{\perp} \mathcal{L}\left(f_{\alpha 0}\right) \\
& \times \mathrm{e}^{i D_{n \alpha} \tau}\left[F_{n \alpha}(\tau)\right]^{(|n|-1)} \sum_{i j} \frac{k_{i} k_{j} \Pi_{i}^{-} \Pi_{j}^{+}}{\left(x_{n}^{-} x_{n}^{+}\right)^{|n|}} \\
& -\frac{k_{\|}^{2}}{k^{2}} \sum_{\alpha} \frac{4 \pi q_{\alpha}^{2}}{m_{\alpha} \omega^{2}} \int d^{3} u \frac{u_{\|}}{\gamma} L\left(f_{\alpha 0}\right)
\end{aligned}
$$

where we did not use the indexes $n \alpha$ in the components $\Pi_{i}$, for simplicity. We see that it is necessary to evaluate the following product,

$$
\begin{gathered}
\sum_{i} k_{i} \Pi_{i}^{ \pm}=k_{\|} \frac{u_{\|}}{u_{\perp}} J_{|n|}\left(x_{n}^{ \pm}\right) F_{n \alpha}^{1 / 2}(\tau) \\
\pm k_{\perp} \cos \psi\left[n J_{|n|}\left(x_{n}^{ \pm}\right) G_{n \alpha}^{ \pm}(\tau)+i \frac{J_{|n|+1}\left(x_{n}^{ \pm}\right)}{x_{n}^{ \pm}} F_{n \alpha}^{1 / 2}(\tau) b_{\alpha} \sin \psi\right] \\
+i k_{\perp} \sin \psi\left[|n| J_{|n|}\left(x_{n}^{ \pm}\right) G_{n \alpha}^{ \pm}(\tau)\right. \\
\left.\mp \frac{J_{|n|+1}\left(x_{n}^{ \pm}\right)}{x_{n}^{ \pm}} F_{n \alpha}^{1 / 2}(\tau)\left(b_{\alpha} \cos \psi \pm \mathcal{K}_{n} \tau\right)\right] \\
= \pm\left(S_{n} k_{\perp} \cos \psi \pm i k_{\perp} \sin \psi\right)\left[|n| J_{|n|}\left(x_{n}^{ \pm}\right) G_{n \alpha}^{ \pm}(\tau)\right]
\end{gathered}
$$




$$
\begin{gathered}
-i k_{\perp} \sin \psi\left[\frac{J_{|n|+1}\left(x_{n}^{ \pm}\right)}{x_{n}^{ \pm}} F_{n \alpha}^{1 / 2}(\tau) \mathcal{K}_{n} \tau\right] \\
+k_{\|} \frac{u_{\|}}{u_{\perp}} J_{|n|}\left(x_{n}^{ \pm}\right) F_{n \alpha}^{1 / 2}(\tau) .
\end{gathered}
$$

Therefore, the quantity of interest is given by

$$
\begin{gathered}
\sum_{j} k_{j} \Pi_{j}^{+} \sum_{i} k_{i} \Pi_{i}^{-} \\
=\left\{\left(S_{n} k_{\perp} \cos \psi+i k_{\perp} \sin \psi\right)\left[|n| J_{|n|}\left(x_{n}^{+}\right) G_{n \alpha}^{+}(\tau)\right]\right. \\
\left.-i k_{\perp} \sin \psi\left[\frac{J_{|n|+1}\left(x_{n}^{+}\right)}{x_{n}^{+}} F_{n \alpha}^{1 / 2}(\tau) \mathcal{K}_{n} \tau\right]+k_{\|} \frac{u_{\|}}{u_{\perp}} J_{|n|}\left(x_{n}^{+}\right) F_{n \alpha}^{1 / 2}(\tau)\right\} \\
\times\left\{-\left(S_{n} k_{\perp} \cos \psi-i k_{\perp} \sin \psi\right)\left[|n| J_{|n|}\left(x_{n}^{-}\right) G_{n \alpha}^{-}(\tau)\right]\right. \\
\left.-i k_{\perp} \sin \psi\left[\frac{J_{|n|+1}\left(x_{n}^{-}\right)}{x_{n}^{-}} F_{n \alpha}^{1 / 2}(\tau) \mathcal{K}_{n} \tau\right]+k_{\|} \frac{u_{\|}}{u_{\perp}} J_{|n|}\left(x_{n}^{-}\right) F_{n \alpha}^{1 / 2}(\tau)\right\}
\end{gathered}
$$

After some straightforward algebra, using $G^{+} G^{-}=-1$ and $F^{1 / 2} F^{1 / 2}=F$, we arrive to the following

$$
\begin{gathered}
\sum_{j} k_{j} \Pi_{j}^{+} \sum_{i} k_{i} \Pi_{i}^{-}=k_{\perp}^{2} n^{2} J_{|n|}\left(x_{n}^{+}\right) J_{|n|}\left(x_{n}^{-}\right) \\
-i k_{\perp}^{2} \sin \psi\left(S_{n} \cos \psi+i \sin \psi\right) \\
\times\left[\frac{|n| J_{|n|}\left(x_{n}^{+}\right) J_{|n|+1}\left(x_{n}^{-}\right)}{x_{n}^{-}} F_{n \alpha}^{1 / 2}(\tau) G_{n \alpha}^{+}(\tau) \mathcal{K}_{n} \tau\right] \\
+k_{||} k_{\perp}\left(S_{n} \cos \psi+i \sin \psi\right) \\
\times \frac{u_{\|}}{u_{\perp}}|n| J_{|n|}\left(x_{n}^{+}\right) J_{|n|}\left(x_{n}^{-}\right) F_{n \alpha}^{1 / 2}(\tau) G_{n \alpha}^{+}(\tau) \\
+i k_{\perp}^{2} \sin \psi\left(S_{n} \cos \psi-i \sin \psi\right) \\
\times\left[\frac{|n| J_{|n|+1}\left(x_{n}^{+}\right) J_{|n|}\left(x_{n}^{-}\right)}{x_{n}^{+}} F_{n \alpha}^{1 / 2}(\tau) G_{n \alpha}^{-}(\tau) \mathcal{K}_{n} \tau\right] \\
-k_{\perp}^{2} \sin ^{2} \psi\left[\frac{J_{|n|+1}\left(x_{n}^{+}\right) J_{|n|+1}\left(x_{n}^{-}\right)}{x_{n}^{+} x_{n}^{-}} F_{n \alpha}(\tau)\left(\mathcal{K}_{n} \tau\right)^{2}\right] \\
-i k_{\|} k_{\perp} \sin \psi \frac{u_{\|}}{u_{\perp}}\left[\frac{J_{|n|+1}\left(x_{n}^{+}\right) J_{|n|}\left(x_{n}^{-}\right)}{x_{n}^{+}} F_{n \alpha}(\tau) \mathcal{K}_{n} \tau\right] \\
-k_{\|} k_{\perp}\left(S_{n} \cos \psi-i \sin \psi\right) \\
+i k_{\|} k_{\perp} \frac{u_{\|}^{2}}{u_{\perp}^{2}} J_{|n|}\left(x_{n}^{+}\right) J_{|n|}\left(x_{n}^{-}\right) F_{n \alpha}(\tau) \\
\times \frac{u_{\|}}{u_{\perp}}\left[|n| J_{|n|}\left(x_{n}^{+}\right) J_{|n|}\left(x_{n}^{-}\right) F_{n \alpha}^{1 / 2}(\tau) G_{n \alpha}^{-}(\tau)\right] \\
u_{\|}\left[\frac{J_{|n|}\left(x_{n}^{+}\right) J_{|n|+1}\left(x_{n}^{-}\right)}{x_{n}^{-}} F_{n \alpha}(\tau) \mathcal{K}_{n} \tau\right]
\end{gathered}
$$

Using now the expression for $F^{1 / 2} G^{ \pm}$, and separating the terms containing $S_{n} \cos \psi$ from those with $i \sin \psi$, we obtain

$$
\sum_{j} k_{j} \Pi_{j}^{+} \sum_{i} k_{i} \Pi_{i}^{-}=k_{\perp}^{2} n^{2} J_{|n|}\left(x_{n}^{+}\right) J_{|n|}\left(x_{n}^{-}\right)
$$

$$
+k_{\|}^{2} \frac{u_{\|}^{2}}{u_{\perp}^{2}} J_{|n|}\left(x_{n}^{+}\right) J_{|n|}\left(x_{n}^{-}\right) F_{n \alpha}(\tau)
$$$$
+i k_{\perp}^{2} S_{n} \sin \psi \cos \psi\left[\frac{|n| J_{|n|}\left(x_{n}^{+}\right) J_{|n|+1}\left(x_{n}^{-}\right)}{x_{n}^{-}}\right.
$$$$
\left.\times\left(\mathcal{K}_{n} \tau-b_{\alpha} \cos \psi+i S_{n} b_{\alpha} \sin \psi\right) \mathcal{K}_{n} \tau\right]
$$$$
+i k_{\perp}^{2} S_{n} \sin \psi \cos \psi\left[\frac{|n| J_{|n|+1}\left(x_{n}^{+}\right) J_{|n|}\left(x_{n}^{-}\right)}{x_{n}^{+}}\right.
$$$$
\left.\times\left(-\mathcal{K}_{n} \tau-b_{\alpha} \cos \psi-i S_{n} b_{\alpha} \sin \psi\right) \mathcal{K}_{n} \tau\right]
$$$$
+k_{\perp}^{2} \sin ^{2} \psi\left[\frac{|n| J_{|n|}\left(x_{n}^{+}\right) J_{|n|+1}\left(x_{n}^{-}\right)}{x_{n}^{-}}\right.
$$

$$
\left.\times\left(-\mathcal{K}_{n} \tau+b_{\alpha} \cos \psi-i S_{n} b_{\alpha} \sin \psi\right) \mathcal{K}_{n} \tau\right]
$$$$
+k_{\perp}^{2} \sin ^{2} \psi\left[\frac{|n| J_{|n|+1}\left(x_{n}^{+}\right) J_{|n|}\left(x_{n}^{-}\right)}{x_{n}^{+}}\right.
$$

$$
\left.\times\left(-\mathcal{K}_{n} \tau-b_{\alpha} \cos \psi-i S_{n} b_{\alpha} \sin \psi\right) \mathcal{K}_{n} \tau\right]
$$

$$
-k_{\perp}^{2} \sin ^{2} \psi\left[\frac{J_{|n|+1}\left(x_{n}^{+}\right) J_{|n|+1}\left(x_{n}^{-}\right)}{x_{n}^{+} x_{n}^{-}} F_{n \alpha}(\tau)\left(\mathcal{K}_{n} \tau\right)^{2}\right]
$$$$
+2 k_{||} k_{\perp} S_{n} \cos \psi \frac{u_{\|}}{u_{\perp}}|n| J_{|n|}\left(x_{n}^{+}\right) J_{|n|}\left(x_{n}^{-}\right)\left(b_{\alpha} \cos \psi\right)
$$

$$
-i 2 k_{\|} k_{\perp} \sin \psi \frac{u_{\|}}{u_{\perp}}|n| J_{|n|}\left(x_{n}^{+}\right) J_{|n|}\left(x_{n}^{-}\right)\left(\mathcal{K}_{n} \tau+i S_{n} b_{\alpha} \sin \psi\right)
$$

$$
-i k_{\|} k_{\perp} \sin \psi \frac{u_{\|}}{u_{\perp}}\left[\frac{J_{|n|+1}\left(x_{n}^{+}\right) J_{|n|}\left(x_{n}^{-}\right)}{x_{n}^{+}} F_{n \alpha}(\tau) \mathcal{K}_{n} \tau\right]
$$




$$
-i k_{\|} k_{\perp} \sin \psi \frac{u_{\|}}{u_{\perp}}\left[\frac{J_{|n|}\left(x_{n}^{+}\right) J_{|n|+1}\left(x_{n}^{-}\right)}{x_{n}^{-}} F_{n \alpha}(\tau) \mathcal{K}_{n} \tau\right] .
$$

This expression must be used along with Eq. (5), which constitutes a general form of the longitudinal dielectric constant. An alternative form can be obtained by use of the following property of Bessel functions,

$$
J_{n+1}(x)=\left(\frac{n J_{n}(x)}{x}-J_{n}^{\prime}(x)\right) .
$$

Using this property, Eq. (6) can be written as follows,

$$
\begin{aligned}
& \sum_{i j} \frac{k_{i} k_{j} \Pi_{i}^{-} \Pi_{j}^{+}}{k^{2}}=\frac{k_{\perp}^{2}}{k^{2}} n^{2} J_{|n|}\left(x_{n}^{+}\right) J_{|n|}\left(x_{n}^{-}\right) \\
& +\frac{k_{\|}^{2}}{k^{2}} \frac{u_{\|}^{2}}{u_{\perp}^{2}} J_{|n|}\left(x_{n}^{+}\right) J_{|n|}\left(x_{n}^{-}\right) F_{n \alpha}(\tau)+i \frac{k_{\perp}^{2}}{k^{2}} S_{n} \sin \psi \cos \psi \\
& \times\left[\left(\frac{|n|^{2} J_{|n|}\left(x_{n}^{+}\right) J_{|n|}\left(x_{n}^{-}\right)}{x_{n}^{-} x_{n}^{-}}-\frac{|n| J_{|n|}\left(x_{n}^{+}\right) J_{|n|}^{\prime}\left(x_{n}^{-}\right)}{x_{n}^{-}}\right)\right. \\
& \left.\times\left(\mathcal{K}_{n} \tau-b_{\alpha} \cos \psi+i S_{n} b_{\alpha} \sin \psi\right)\left(\mathcal{K}_{n} \tau\right)\right]+i \frac{k_{\perp}^{2}}{k^{2}} S_{n} \sin \psi \cos \psi \\
& \times\left[\left(\frac{|n|^{2} J_{|n|}\left(x_{n}^{+}\right) J_{|n|}\left(x_{n}^{-}\right)}{x_{n}^{+} x_{n}^{+}}-\frac{|n| J_{|n|}\left(x_{n}^{-}\right) J_{|n|}^{\prime}\left(x_{n}^{+}\right)}{x_{n}^{+}}\right)\right. \\
& \left.\times\left(-\mathcal{K}_{n} \tau-b_{\alpha} \cos \psi-i S_{n} b_{\alpha} \sin \psi\right)\left(\mathcal{K}_{n} \tau\right)\right] \\
& +\frac{k_{\perp}^{2}}{k^{2}} \sin ^{2} \psi\left[\left(\frac{|n|^{2} J_{|n|}\left(x_{n}^{+}\right) J_{|n|}\left(x_{n}^{-}\right)}{x_{n}^{-} x_{n}^{-}}-\frac{|n| J_{|n|}\left(x_{n}^{+}\right) J_{|n|}^{\prime}\left(x_{n}^{-}\right)}{x_{n}^{-}}\right)\right. \\
& \left.\times\left(-\mathcal{K}_{n} \tau+b_{\alpha} \cos \psi-i S_{n} b_{\alpha} \sin \psi\right)\left(\mathcal{K}_{n} \tau\right)\right] \\
& +\frac{k_{\perp}^{2}}{k^{2}} \sin ^{2} \psi\left[\left(\frac{|n|^{2} J_{|n|}\left(x_{n}^{+}\right) J_{|n|}\left(x_{n}^{-}\right)}{x_{n}^{+} x_{n}^{+}}-\frac{|n| J_{|n|}\left(x_{n}^{-}\right) J_{|n|}^{\prime}\left(x_{n}^{+}\right)}{x_{n}^{+}}\right)\right. \\
& \left.\times\left(-\mathcal{K}_{n} \tau-b_{\alpha} \cos \psi-i S_{n} b_{\alpha} \sin \psi\right)\left(\mathcal{K}_{n} \tau\right)\right] \\
& -\frac{k_{\perp}^{2}}{k^{2}} \sin ^{2} \psi\left[\frac{1}{x_{n}^{+} x_{n}^{-}}\left(\frac{|n| J_{|n|}\left(x_{n}^{+}\right)}{x_{n}^{+}}-J_{|n|}^{\prime}\left(x_{n}^{+}\right)\right)\right. \\
& \left.\left(\frac{|n| J_{|n|}\left(x_{n}^{-}\right)}{x_{n}^{-}}-J_{|n|}^{\prime}\left(x_{n}^{-}\right)\right) F_{n \alpha}(\tau)\left(\mathcal{K}_{n} \tau\right)^{2}\right] \\
& +\frac{2 k_{\|} k_{\perp}}{k^{2}} S_{n} \cos \psi \frac{u_{\|}}{u_{\perp}}|n| J_{|n|}\left(x_{n}^{+}\right) J_{|n|}\left(x_{n}^{-}\right)\left(b_{\alpha} \cos \psi\right) \\
& -i \frac{2 k_{\|} k_{\perp}}{k^{2}} \sin \psi \frac{u_{\|}}{u_{\perp}}|n| J_{|n|}\left(x_{n}^{+}\right) J_{|n|}\left(x_{n}^{-}\right)\left(\mathcal{K}_{n} \tau+i S_{n} b_{\alpha} \sin \psi\right) \\
& -i \frac{k_{\|} k_{\perp}}{k^{2}} \sin \psi \frac{u_{\|}}{u_{\perp}}\left[\left(\frac{|n| J_{|n|}\left(x_{n}^{+}\right) J_{|n|}\left(x_{n}^{-}\right)}{x_{n}^{+} x_{n}^{+}}-\frac{J_{|n|}\left(x_{n}^{-}\right) J_{|n|}^{\prime}\left(x_{n}^{+}\right)}{x_{n}^{+}}\right)\right. \\
& \left.\times F_{n \alpha}(\tau)\left(\mathcal{K}_{n} \tau\right)\right] \\
& -i \frac{k_{\|} k_{\perp}}{k^{2}} \sin \psi \frac{u_{\|}}{u_{\perp}}\left[\left(\frac{|n| J_{|n|}\left(x_{n}^{+}\right) J_{|n|}\left(x_{n}^{-}\right)}{x_{n}^{-} x_{n}^{-}}-\frac{J_{|n|}\left(x_{n}^{+}\right) J_{|n|}^{\prime}\left(x_{n}^{-}\right)}{x_{n}^{-}}\right)\right. \\
& \left.\times F_{n \alpha}(\tau)\left(\mathcal{K}_{n} \tau\right)\right] .
\end{aligned}
$$


This is an alternative expression to be utilized instead of Eq. (6), for evaluation of the dielectric constant, given by Eq. (5).

In the homogeneous limit, $\mathcal{K}_{n} \rightarrow 0, x_{n}^{ \pm} \rightarrow b_{\alpha}$, and $F_{n \alpha} \rightarrow b_{\alpha}^{2}$. In that case it is easy to show that Eq. (7) is reduced to the following expression,

$$
\sum_{i j} \frac{k_{i} k_{j} \Pi_{i}^{-} \Pi_{j}^{+}}{k^{2}} \rightarrow b_{\alpha}^{2} J_{|n|}^{2}\left(b_{\alpha}\right)\left[\frac{k_{\perp}}{k} \frac{n}{b_{\alpha}}+\frac{k_{\|}}{k} \frac{u_{\|}}{u_{\perp}}\right]^{2}
$$

and therefore,

$$
\begin{aligned}
\varepsilon_{l}= & 1+\sum_{\alpha} \frac{4 \pi q_{\alpha}^{2}}{m_{\alpha} \omega} \sum_{n \rightarrow-\infty}^{\infty} \int d^{3} u u_{\perp} \mathcal{L}\left(f_{\alpha 0}\right) \\
& \times \frac{1}{D_{n \alpha}} J_{|n|}^{2}\left(b_{\alpha}\right)\left[\frac{k_{\perp}}{k} \frac{n}{b_{\alpha}}+\frac{k_{\|}}{k} \frac{u_{\|}}{u_{\perp}}\right]^{2} \\
& -\frac{k_{\|}^{2}}{k^{2}} \sum_{\alpha} \frac{4 \pi q_{\alpha}^{2}}{m_{\alpha} \omega^{2}} \int d^{3} u \frac{u_{\|}}{\gamma} L\left(f_{\alpha 0}\right),
\end{aligned}
$$

where we have performed the integration over the time variable,

$$
\int_{0}^{\infty} d \tau \mathrm{e}^{i D_{n \alpha} \tau}=\frac{i}{D_{n \alpha}} .
$$

Equation (8) corresponds to the homogeneous contribution appearing in Ref. [2]. It is easy to see that the same limit is obtained using the alternative form, based on Eq. (7).

Another interesting limit to be considered corresponds to the case of propagation parallel to the magnetic field, $b_{\alpha} \rightarrow 0$, where

$$
\begin{gathered}
x_{n}^{ \pm} \rightarrow\left|\mathcal{K}_{n}\right| \tau, \\
F_{n \alpha} \rightarrow-\left(\mathcal{K}_{n} \tau\right)^{2}=-x_{n}^{+} x_{n}^{-} .
\end{gathered}
$$

The Bessel functions become all independent of $k_{\perp}$. Therefore, for vanishing $k_{\perp}$, all terms which have combinations of Bessel functions multiplied by $k_{\perp}$ should vanish. After a small amount of algebra, it is easy to show that Eq. (5) becomes

$$
\begin{aligned}
\varepsilon_{l}=1-i \sum_{\alpha} & \frac{4 \pi q_{\alpha}^{2}}{m_{\alpha} \omega} \sum_{n \rightarrow-\infty}^{\infty}(-1)^{|n|} \int_{0}^{\infty} d \tau \int d^{3} u u_{\perp} \mathcal{L}\left(f_{\alpha 0}\right) \\
& \times \mathrm{e}^{i D_{n \alpha} \tau} \frac{u_{\|}^{2}}{u_{\perp}^{2}} J_{|n|}\left(x_{n}^{+}\right) J_{|n|}\left(x_{n}^{-}\right) \\
& -\sum_{\alpha} \frac{4 \pi q_{\alpha}^{2}}{m_{\alpha} \omega^{2}} \int d^{3} u \frac{u_{\|}}{\gamma} L\left(f_{\alpha 0}\right) .
\end{aligned}
$$

For propagation parallel to the direction of the inhomogeneity,

$$
\begin{gathered}
x_{n}^{ \pm}=\left[b_{\alpha}^{2} \pm 2 b_{\alpha} \mathcal{K}_{n} \tau+\mathcal{K}_{n}^{2} \tau^{2}\right]^{1 / 2} \\
=\left[\left(b_{\alpha} \pm \mathcal{K}_{n} \tau\right)^{2}\right]^{1 / 2}=\left|b_{\alpha} \pm \mathcal{K}_{n} \tau\right|, \\
F_{n \alpha}(\tau)=b_{\alpha}^{2}-\left(\mathcal{K}_{n} \tau\right)^{2},
\end{gathered}
$$

and

$$
\begin{gathered}
\varepsilon_{l}=1-i \sum_{\alpha} \frac{4 \pi q_{\alpha}^{2}}{m_{\alpha} \omega} \sum_{n \rightarrow-\infty}^{\infty} \int_{0}^{\infty} d \tau \int d^{3} u u_{\perp} \mathcal{L}\left(f_{\alpha 0}\right) \\
\times \mathrm{e}^{i D_{n \alpha} \tau} \frac{\left[F_{n \alpha}(\tau)\right]^{(|n|-1)}}{\left(x_{n}^{-} x_{n}^{+}\right)^{|n|}} n^{2} J_{|n|}\left(x_{n}^{+}\right) J_{|n|}\left(x_{n}^{-}\right)
\end{gathered}
$$

The last limiting case to be considered is the case of propagation perpendicular to the direction of the magnetic field and of the inhomogeneity.

$$
\begin{gathered}
x_{n}^{ \pm}=\left[b_{\alpha}^{2}+\mathcal{K}_{n}^{2} \tau^{2}\right]^{1 / 2}, \\
F_{n \alpha}(\tau)=b_{\alpha}^{2}-\left(\mathcal{K}_{n} \tau\right)^{2}-i 2 S_{n} b_{\alpha}\left(\mathcal{K}_{n} \tau\right),
\end{gathered}
$$

The dielectric constant in these cases is given by Eq. (5), with

$$
\begin{gathered}
\sum_{i j} \frac{k_{i} k_{j} \Pi_{i}^{-} \Pi_{j}^{+}}{k^{2}}=n^{2} J_{|n|}\left(x_{n}^{+}\right) J_{|n|}\left(x_{n}^{-}\right) \\
-\left[\left(\frac{|n|^{2} J_{|n|}\left(x_{n}^{+}\right) J_{|n|}\left(x_{n}^{-}\right)}{x_{n}^{-} x_{n}^{-}}-\frac{|n| J_{|n|}\left(x_{n}^{+}\right) J_{|n|}^{\prime}\left(x_{n}^{-}\right)}{x_{n}^{-}}\right)\right.
\end{gathered}
$$

$$
\begin{gathered}
\left.\times\left(\mathcal{K}_{n} \tau+i S_{n} b_{\alpha}\right)\left(\mathcal{K}_{n} \tau\right)\right] \\
-\left[\left(\frac{|n|^{2} J_{|n|}\left(x_{n}^{+}\right) J_{|n|}\left(x_{n}^{-}\right)}{x_{n}^{+} x_{n}^{+}}-\frac{|n| J_{|n|}\left(x_{n}^{-}\right) J_{|n|}^{\prime}\left(x_{n}^{+}\right)}{x_{n}^{+}}\right)\right. \\
\left.\times\left(\mathcal{K}_{n} \tau+i S_{n} b_{\alpha}\right)\left(\mathcal{K}_{n} \tau\right)\right] \\
-\left[\frac{1}{x_{n}^{+} x_{n}^{-}}\left(\frac{|n| J_{|n|}\left(x_{n}^{+}\right)}{x_{n}^{+}}-J_{|n|}^{\prime}\left(x_{n}^{+}\right)\right)\right. \\
\left.\times\left(\frac{|n| J_{|n|}\left(x_{n}^{-}\right)}{x_{n}^{-}}-J_{|n|}^{\prime}\left(x_{n}^{-}\right)\right) F_{n \alpha}(\tau)\left(\mathcal{K}_{n} \tau\right)^{2}\right] .
\end{gathered}
$$


In the same limit, an alternative expression can be obtained using Eq. (7), resulting the following,

$$
\begin{gathered}
\sum_{i j} \frac{k_{i} k_{j} \Pi_{i}^{-} \Pi_{j}^{+}}{k^{2}} \rightarrow n^{2} J_{|n|}\left(x_{n}^{+}\right) J_{|n|}\left(x_{n}^{-}\right) \\
-\left[\frac{|n| J_{|n|}\left(x_{n}^{+}\right) J_{|n|+1}\left(x_{n}^{-}\right)}{x_{n}^{-}}\left(\mathcal{K}_{n} \tau+i S_{n} b_{\alpha}\right)\left(\mathcal{K}_{n} \tau\right)\right] \\
-\left[\frac{|n| J_{|n|+1}\left(x_{n}^{+}\right) J_{|n|}\left(x_{n}^{-}\right)}{x_{n}^{+}}\left(\mathcal{K}_{n} \tau+i S_{n} b_{\alpha}\right)\left(\mathcal{K}_{n} \tau\right)\right] \\
-\left[\frac{J_{|n|+1}\left(x_{n}^{+}\right) J_{|n|+1}\left(x_{n}^{-}\right)}{x_{n}^{+} x_{n}^{-}} F_{n \alpha}(\tau)\left(\mathcal{K}_{n} \tau\right)^{2}\right]
\end{gathered}
$$

\section{The dielectric constant for the case} of a Maxwellian distribution func-

\section{tion}

Let us assume a Maxwellian distribution function for particles of type $\alpha$,

$$
\begin{gathered}
f_{\alpha 0}=\frac{n_{\alpha} \mu_{\alpha}^{3 / 2}}{(2 \pi)^{3 / 2}} e^{-\mu_{\alpha} u^{2} / 2}, \quad \mu_{\alpha}=\frac{m_{\alpha} c^{2}}{T_{\alpha}} \\
\frac{\partial f_{\alpha 0}}{\partial u_{\perp}}=-\mu_{\alpha} u_{\perp} f_{\alpha 0}
\end{gathered}
$$

Choosing the coordinate system in order to have $x=0$, and considering, for simplicity, the non-relativistic limit, we obtain

$$
\begin{gathered}
\varepsilon_{l}=1+i \sum_{\alpha} \frac{\omega_{\alpha}^{2}}{\omega} \frac{\mu_{\alpha}^{5 / 2}}{(2 \pi)^{1 / 2}} \sum_{n \rightarrow-\infty}^{\infty} \int_{0}^{\infty} d \tau \mathrm{e}^{i\left(\omega-n \Omega_{\alpha}\right) \tau} \\
\quad \times \int_{-\infty}^{\infty} d u_{\|} e^{-\mu_{\alpha} u_{\|}^{2} / 2} e^{-i c k_{\|} u_{\|} \tau} \\
\times \int_{0}^{\infty} d u_{\perp} u_{\perp}^{3} e^{-\mu_{\alpha} u_{\perp}^{2} / 2} e^{-i \epsilon_{B} k_{\perp} u_{\perp}^{2} c^{2} \sin \psi \tau /\left(2 \Omega_{\alpha}\right)}
\end{gathered}
$$

$$
\times \frac{\left[F_{n \alpha}(\tau)\right]^{(|n|-1)}}{\left(x_{n}^{-} x_{n}^{+}\right)^{|n|}} \sum_{i j} \frac{k_{i} k_{j} \Pi_{i}^{-} \Pi_{j}^{+}}{k^{2}} .
$$

The quantities $x_{n}^{ \pm}$and $F_{n \alpha}$ do not depend on the parallel momentum, which only appears in the product $\Pi_{i}^{-} \Pi_{j}^{+}$. Therefore we are left with the following integrals over parallel momentum,

$$
I_{j}=\int_{-\infty}^{\infty} d u_{\|} e^{-\mu_{\alpha} u_{\|}^{2} / 2} e^{-i c k_{\|} u_{\|} \tau}\left(u_{\|}\right)^{j},
$$

with $j=0,1,2$. These can be easily performed, with the following results,

$$
\begin{gathered}
j=0: \quad I_{0}=\frac{(2 \pi)^{1 / 2}}{\mu_{\alpha}^{1 / 2}} e^{-c^{2} k_{\|}^{2} \tau^{2} /\left(2 \mu_{\alpha}\right)} \\
j=1: \quad I_{1}=-i \frac{(2 \pi)^{1 / 2}}{\mu_{\alpha}^{1 / 2}} \frac{c k_{\|} \tau}{\mu_{\alpha}} e^{-c^{2} k_{\|}^{2} \tau^{2} /\left(2 \mu_{\alpha}\right)} \\
j=2: \quad I_{2}=\frac{(2 \pi)^{1 / 2}}{\mu_{\alpha}^{1 / 2}} \frac{1}{\mu_{\alpha}}\left(1-\frac{c^{2} k_{\|}^{2} \tau^{2}}{\mu_{\alpha}}\right) e^{-c^{2} k_{\|}^{2} \tau^{2} /\left(2 \mu_{\alpha}\right)}
\end{gathered}
$$

Using these results,

$$
\begin{gathered}
\varepsilon_{l}=1+i \sum_{\alpha} \frac{\omega_{\alpha}^{2}}{\omega} \frac{\mu_{\alpha}^{5 / 2}}{(2 \pi)^{1 / 2}} \frac{(2 \pi)^{1 / 2}}{\mu_{\alpha}^{1 / 2}} \\
\times \sum_{n \rightarrow-\infty}^{\infty} \int_{0}^{\infty} d \tau \mathrm{e}^{i\left(\omega-n \Omega_{\alpha}\right) \tau} e^{-c^{2} k_{\|}^{2} \tau^{2} /\left(2 \mu_{\alpha}\right)} \\
\times \int_{0}^{\infty} d u_{\perp} u_{\perp}^{3} e^{-\mu_{\alpha} u_{\perp}^{2} / 2} e^{-i \epsilon_{B} k_{\perp} u_{\perp}^{2} c^{2} \sin \psi \tau /\left(2 \Omega_{\alpha}\right)} \\
\times \frac{\left[F_{n \alpha}(\tau)\right]^{(|n|-1)}}{\left(x_{n}^{-} x_{n}^{+}\right)^{|n|}} \Lambda
\end{gathered}
$$

where

$$
\begin{gathered}
\Lambda=\frac{k_{\perp}^{2}}{k^{2}} n^{2} J_{|n|}\left(x_{n}^{+}\right) J_{|n|}\left(x_{n}^{-}\right) \\
+\frac{k_{\|}^{2}}{k^{2}} \frac{1}{u_{\perp}^{2}} \frac{1}{\mu_{\alpha}}\left(1-\frac{c^{2} k_{\|}^{2} \tau^{2}}{\mu_{\alpha}}\right) J_{|n|}\left(x_{n}^{+}\right) J_{|n|}\left(x_{n}^{-}\right) F_{n \alpha}(\tau) \\
+i \frac{k_{\perp}^{2}}{k^{2}} S_{n} \sin \psi \cos \psi\left[\frac{|n| J_{|n|}\left(x_{n}^{+}\right) J_{|n|+1}\left(x_{n}^{-}\right)}{x_{n}^{-}}\right. \\
\left.\times\left(\mathcal{K}_{n} \tau-b_{\alpha} \cos \psi+i S_{n} b_{\alpha} \sin \psi\right) \mathcal{K}_{n} \tau\right] \\
+i \frac{k_{\perp}^{2}}{k^{2}} S_{n} \sin \psi \cos \psi\left[\frac{|n| J_{|n|+1}\left(x_{n}^{+}\right) J_{|n|}\left(x_{n}^{-}\right)}{x_{n}^{+}}\right. \\
\left.\times\left(-\mathcal{K}_{n} \tau-b_{\alpha} \cos \psi-i S_{n} b_{\alpha} \sin \psi\right) \mathcal{K}_{n} \tau\right]
\end{gathered}
$$




$$
\begin{gathered}
+\frac{k_{\perp}^{2}}{k^{2}} \sin ^{2} \psi\left[\frac{|n| J_{|n|}\left(x_{n}^{+}\right) J_{|n|+1}\left(x_{n}^{-}\right)}{x_{n}^{-}}\right. \\
\left.\times\left(-\mathcal{K}_{n} \tau+b_{\alpha} \cos \psi-i S_{n} b_{\alpha} \sin \psi\right) \mathcal{K}_{n} \tau\right] \\
+\frac{k_{\perp}^{2}}{k^{2}} \sin ^{2} \psi\left[\frac{|n| J_{|n|+1}\left(x_{n}^{+}\right) J_{|n|}\left(x_{n}^{-}\right)}{x_{n}^{+}}\right. \\
\left.\times\left(-\mathcal{K}_{n} \tau-b_{\alpha} \cos \psi-i S_{n} b_{\alpha} \sin \psi\right) \mathcal{K}_{n} \tau\right] \\
-\frac{k_{\perp}^{2}}{k^{2}} \sin ^{2} \psi\left[\frac{J_{|n|+1}\left(x_{n}^{+}\right) J_{|n|+1}\left(x_{n}^{-}\right)}{x_{n}^{+} x_{n}^{-}} F_{n \alpha}(\tau)\left(\mathcal{K}_{n} \tau\right)^{2}\right] \\
+\frac{2 k_{\|} k_{\perp}}{k^{2}} S_{n} \cos \psi \frac{1}{u_{\perp}}\left(-i \frac{c k_{||} \tau}{\mu_{\alpha}}\right) \\
\times|n| J_{|n|}\left(x_{n}^{+}\right) J_{|n|}\left(x_{n}^{-}\right)\left(b_{\alpha} \cos \psi\right) \\
-i \frac{2 k_{||} k_{\perp}}{k^{2}} \sin \psi \frac{1}{u_{\perp}}\left(-i \frac{c k_{||} \tau}{\mu_{\alpha}}\right)|n| J_{|n|}\left(x_{n}^{+}\right) J_{|n|}\left(x_{n}^{-}\right) \\
\times i \frac{k_{||} k_{\perp}}{k^{2}} \sin \psi \frac{1}{u_{\perp}}\left(-i \frac{c k_{||} \tau}{\mu_{\alpha}}\right)\left[\frac{J_{|n|}\left(x_{n}^{+}\right) J_{|n|+1}\left(x_{n}^{-}\right)}{x_{n}^{-}} F_{n \alpha}(\tau) \mathcal{K}_{n} \tau\right] \\
-i \frac{k_{||} k_{\perp}}{k^{2}} \sin \psi \frac{1}{u_{\perp}}\left(-i \frac{c k_{\|} \tau}{\mu_{\alpha}}\right)\left[\frac{J_{|n|+1}\left(x_{n}^{+}\right) J_{|n|}\left(x_{n}^{-}\right)}{x_{n}^{+}} F_{n \alpha}(\tau) \mathcal{K}_{n} \tau\right]
\end{gathered}
$$

In order to simplify the ensuing calculations, we introduce the following notation,

$$
\begin{gathered}
x_{n}^{ \pm} \equiv y_{n}^{ \pm} u_{\perp}, \\
F_{n \alpha}(\tau) \equiv f_{n \alpha}(\tau) u_{\perp}^{2},
\end{gathered}
$$

where

$$
\begin{gathered}
y_{n}^{ \pm}=\left[c^{2} k_{\perp}^{2} / \Omega_{\alpha}^{2} \pm 2\left(c k_{\perp} / \Omega_{\alpha}\right) \cos \psi\left(n c \epsilon_{B} / 2\right) \tau\right. \\
\left.+\left(n^{2} \epsilon_{B}^{2} c^{2} / 4\right) \tau^{2}\right]^{1 / 2}, \\
f_{n \alpha}(\tau)=\left[c^{2} k_{\perp}^{2} / \Omega_{\alpha}^{2}-\left(n^{2} \epsilon_{B}^{2} c^{2} / 4\right) \tau^{2}\right. \\
\left.\left.-i 2\left(c k_{\perp} / \Omega_{\alpha}\right) \sin (\psi)\left(|n| c \epsilon_{B} / 2\right) \tau\right)\right] .
\end{gathered}
$$

Moreover, we define

$$
N_{\perp}=\frac{c k_{\perp}}{\omega}, \quad N_{\|}=\frac{c k_{\|}}{\omega}, \quad N_{B}=\frac{c \epsilon_{B}}{\omega}, \quad Y_{\alpha}=\frac{\Omega_{\alpha}}{\omega},
$$

and

$$
\zeta_{\alpha}=N_{B} N_{\perp} \sin \psi / Y_{\alpha}, \quad t=\left(\omega \tau / \mu_{\alpha}\right) .
$$

Using this notation,

$$
\mathcal{K}_{n} \tau=\left(n N_{B} \mu_{\alpha} t / 2\right) u_{\perp}, \quad b_{\alpha}=\left(N_{\perp} / Y_{\alpha}\right) u_{\perp},
$$

and the dielectric constant becomes the following

$$
\varepsilon_{l}=1+i \sum_{\alpha} \frac{\omega_{\alpha}^{2}}{\omega^{2}} \mu_{\alpha}^{3} \sum_{n \rightarrow-\infty}^{\infty} \int_{0}^{\infty} d t \mathrm{e}^{i \mu_{\alpha}\left(1-n Y_{\alpha}\right) t} e^{-\mu_{\alpha} N_{\|}^{2} t^{2} / 2}
$$

$$
\times \frac{\left[f_{n \alpha}(\tau)\right]^{(|n|-1)}}{\left(y_{n}^{-} y_{n}^{+}\right)^{|n|}} \int_{0}^{\infty} d u_{\perp} u_{\perp} e^{-\left(\mu_{\alpha} u_{\perp}^{2} / 2\right)\left[1+i \zeta_{\alpha} t\right]} \Lambda
$$

where

$$
\Lambda=\frac{k_{\perp}^{2}}{k^{2}} n^{2} J_{|n|}\left(x_{n}^{+}\right) J_{|n|}\left(x_{n}^{-}\right)
$$

$$
\begin{gathered}
+\frac{k_{\|}^{2}}{k^{2}} \frac{1}{\mu_{\alpha}}\left(1-\mu_{\alpha} N_{\|}^{2} t^{2}\right) J_{|n|}\left(x_{n}^{+}\right) J_{|n|}\left(x_{n}^{-}\right) f_{n \alpha}(\tau) \\
+i \frac{k_{\perp}^{2}}{k^{2}} S_{n} \sin \psi \cos \psi\left[\frac{|n| J_{|n|}\left(x_{n}^{+}\right) J_{|n|+1}\left(x_{n}^{-}\right)}{y_{n}^{-}}\right. \\
\times\left(\left(n N_{B} \mu_{\alpha} t / 2\right)-\left(N_{\perp} / Y_{\alpha}\right) \cos \psi\right.
\end{gathered}
$$

$$
\left.\left.+i S_{n}\left(N_{\perp} / Y_{\alpha}\right) \sin \psi\right)\left(n N_{B} \mu_{\alpha} t / 2\right) u_{\perp}\right]
$$

$+i \frac{k_{\perp}^{2}}{k^{2}} S_{n} \sin \psi \cos \psi\left[\frac{|n| J_{|n|+1}\left(x_{n}^{+}\right) J_{|n|}\left(x_{n}^{-}\right)}{y_{n}^{+}}\right.$

$$
\begin{gathered}
\times\left(-\left(n N_{B} \mu_{\alpha} t / 2\right)-\left(N_{\perp} / Y_{\alpha}\right) \cos \psi\right. \\
\left.\left.-i S_{n}\left(N_{\perp} / Y_{\alpha}\right) \sin \psi\right)\left(n N_{B} \mu_{\alpha} t / 2\right) u_{\perp}\right] \\
+\frac{k_{\perp}^{2}}{k^{2}} \sin ^{2} \psi\left[\frac{|n| J_{|n|}\left(x_{n}^{+}\right) J_{|n|+1}\left(x_{n}^{-}\right)}{y_{n}^{-}}\right. \\
\times\left(-\left(n N_{B} \mu_{\alpha} t / 2\right)+\left(N_{\perp} / Y_{\alpha}\right) \cos \psi\right. \\
\left.\left.-i S_{n}\left(N_{\perp} / Y_{\alpha}\right) \sin \psi\right)\left(n N_{B} \mu_{\alpha} t / 2\right) u_{\perp}\right]
\end{gathered}
$$




$$
\begin{gathered}
+\frac{k_{\perp}^{2}}{k^{2}} \sin ^{2} \psi\left[\frac{|n| J_{|n|+1}\left(x_{n}^{+}\right) J_{|n|}\left(x_{n}^{-}\right)}{y_{n}^{+}}\right. \\
\times\left(-\left(n N_{B} \mu_{\alpha} t / 2\right)-\left(N_{\perp} / Y_{\alpha}\right) \cos \psi\right. \\
\left.\left.-i S_{n}\left(N_{\perp} / Y_{\alpha}\right) \sin \psi\right)\left(n N_{B} \mu_{\alpha} t / 2\right) u_{\perp}\right] \\
-\frac{k_{\perp}^{2}}{k^{2}} \sin ^{2} \psi\left[\frac{J_{|n|+1}\left(x_{n}^{+}\right) J_{|n|+1}\left(x_{n}^{-}\right)}{y_{n}^{+} y_{n}^{-}}\right. \\
\left.\quad \times f_{n \alpha}(\tau)\left(n N_{B} \mu_{\alpha} t / 2\right)^{2} u_{\perp}^{2}\right] \\
+\frac{2 k_{\|} k_{\perp}}{k^{2}} S_{n} \cos \psi\left(-i N_{\|} t\right)|n| J_{|n|}\left(x_{n}^{+}\right) J_{|n|}\left(x_{n}^{-}\right) \\
\quad \times\left(\left(N_{\perp} / Y_{\alpha}\right) \cos \psi\right) \\
-i \frac{2 k_{\|} k_{\perp}}{k^{2}} \sin \psi\left(-i N_{\|} t\right)|n| J_{|n|}\left(x_{n}^{+}\right) J_{|n|}\left(x_{n}^{-}\right) \\
\times\left(\left(n N_{B} \mu_{\alpha} t / 2\right)+i S_{n}\left(N_{\perp} / Y_{\alpha}\right) \sin \psi\right) \\
-i \frac{k_{\|} k_{\perp}}{k^{2}} \sin \psi\left(-i N_{\|} t\right)\left[\frac{J_{|n|+1}\left(x_{n}^{+}\right) J_{|n|}\left(x_{n}^{-}\right)}{y_{n}^{+}}\right.
\end{gathered}
$$

$$
\begin{gathered}
\left.\times f_{n \alpha}(\tau)\left(n N_{B} \mu_{\alpha} t / 2\right) u_{\perp}\right] \\
-i \frac{k_{\|} k_{\perp}}{k^{2}} \sin \psi\left(-i N_{\|} t\right)\left[\frac{J_{|n|}\left(x_{n}^{+}\right) J_{|n|+1}\left(x_{n}^{-}\right)}{y_{n}^{-}}\right. \\
\left.\times f_{n \alpha}(\tau)\left(n N_{B} \mu_{\alpha} t / 2\right) u_{\perp}\right] .
\end{gathered}
$$

In this expression we can find the following integrals over the perpendicular variable,

$$
\begin{gathered}
I_{\perp 1}=\int_{0}^{\infty} d u_{\perp} u_{\perp} e^{-\left(\mu_{\alpha} u_{\perp}^{2} / 2\right)\left[1+i \zeta_{\alpha} t\right]} J_{|n|}\left(x_{n}^{+}\right) J_{|n|}\left(x_{n}^{-}\right) \\
I_{\perp 2}^{ \pm}=\int_{0}^{\infty} d u_{\perp} u_{\perp}^{2} e^{-\left(\mu_{\alpha} u_{\perp}^{2} / 2\right)\left[1+i \zeta_{\alpha} t\right]} J_{|n|}\left(x_{n}^{\mp}\right) J_{|n|+1}\left(x_{n}^{ \pm}\right) \\
I_{\perp 3}=\int_{0}^{\infty} d u_{\perp} u_{\perp}^{3} e^{-\left(\mu_{\alpha} u_{\perp}^{2} / 2\right)\left[1+i \zeta_{\alpha} t\right]} J_{|n|+1}\left(x_{n}^{+}\right) J_{|n|+1}\left(x_{n}^{-}\right)
\end{gathered}
$$

These integrals can be easily solved, and are given by simple expressions involving the modified Bessel function $I_{n}[9]$

$$
\begin{aligned}
I_{\perp 1}= & \frac{\mathrm{e}^{-\left(\nu_{\alpha}^{2}+\chi_{n \alpha}^{2} t^{2}\right) /\left(1+i \zeta_{\alpha} t\right)}}{\mu_{\alpha}\left(1+i \zeta_{\alpha} t\right)} I_{|n|}\left(\frac{S_{n \alpha}(t)}{1+i \zeta_{\alpha} t}\right) \\
I_{\perp 2}^{ \pm}= & \frac{\mathrm{e}^{-\left(\nu_{\alpha}^{2}+\chi_{n \alpha}^{2} t^{2}\right) /\left(1+i \zeta_{\alpha} t\right)}}{\mu_{\alpha}^{3 / 2}\left(1+i \zeta_{\alpha} t\right)^{2}} \\
& \times\left[T_{n}^{ \pm} I_{|n|}\left(\frac{S_{n \alpha}(t)}{1+i \zeta_{\alpha} t}\right)-T_{n}^{\mp} I_{|n|+1}\left(\frac{S_{n \alpha}(t)}{1+i \zeta_{\alpha} t}\right)\right] \\
I_{\perp 3}= & 2 \frac{\mathrm{e}^{-\left(\nu_{\alpha}^{2}+\chi_{n \alpha}^{2} t^{2}\right) /\left(1+i \zeta_{\alpha} t\right)}}{\mu_{\alpha}^{2}\left(1+i \zeta_{\alpha} t\right)^{2}}\left[\frac{S_{n \alpha}(t)}{1+i \zeta_{\alpha} t} I_{|n|}\left(\frac{S_{n \alpha}(t)}{1+i \zeta_{\alpha} t}\right)\right. \\
& \left.-\left(|n|+\frac{\nu_{\alpha}^{2}+\chi_{n \alpha}^{2} t^{2}}{1+i \zeta_{\alpha} t}\right) I_{|n|+1}\left(\frac{S_{n \alpha}(t)}{1+i \zeta_{\alpha} t}\right)\right],
\end{aligned}
$$

where

$$
\begin{gathered}
T_{n}^{ \pm}=\sqrt{\nu_{\alpha}^{2} \pm 2 \nu_{\alpha} \cos \psi \chi_{n \alpha} t+\chi_{n \alpha}^{2} t^{2}} \\
S_{n \alpha}=\sqrt{\nu_{\alpha}^{4}-2 \nu_{\alpha}^{2} \cos (2 \psi) \chi_{n \alpha}^{2} t^{2}+\chi_{n \alpha}^{4} t^{4}} \\
\chi_{n \alpha}=\frac{n N_{B} \mu_{\alpha}^{1 / 2}}{2}, \\
\nu_{\alpha}=\frac{N_{\perp}}{\mu_{\alpha}^{1 / 2} Y_{\alpha}}=\frac{k_{\perp} v_{\alpha}}{\Omega_{\alpha}}, \quad v_{\alpha}=\left(\frac{T_{\alpha}}{m_{\alpha}}\right)^{1 / 2} .
\end{gathered}
$$

Using the symbols introduced by Eqs. (19), and the notation introduced by Eqs. (21), we can write

$$
f_{n \alpha}=\mu_{\alpha} H_{n \alpha}(t)
$$

where

$$
H_{n \alpha}(t)=\nu_{\alpha}^{2}-i 2 S_{n} \nu_{\alpha} \sin (\psi) \chi_{n \alpha} t-\chi_{n \alpha}^{2} t^{2},
$$

and

$$
\begin{gathered}
y_{n}^{ \pm}=\mu_{\alpha}^{1 / 2} T_{n}^{ \pm}, \\
y_{n}^{+} y_{n}^{-}=\mu_{\alpha} S_{n \alpha}(t) .
\end{gathered}
$$

The dielectric constant therefore can be written as follows,

$\varepsilon_{l}=1+i \sum_{\alpha} \frac{\omega_{\alpha}^{2}}{\omega^{2}} \mu_{\alpha}^{2} \sum_{n \rightarrow-\infty}^{\infty} \int_{0}^{\infty} d t \mathrm{e}^{i \mu_{\alpha}\left(1-n Y_{\alpha}\right) t} e^{-\mu_{\alpha} N_{\|}^{2} t^{2} / 2}$

$$
\times \frac{\left[H_{n \alpha}(t)\right]^{(|n|-1)}}{\left(S_{n \alpha}(t)\right)^{|n|}} \Lambda^{*},
$$

where

$$
\Lambda^{*}=\frac{k_{\perp}^{2}}{k^{2}} n^{2} I_{\perp 1}+\frac{k_{\|}^{2}}{k^{2}}\left(1-\mu_{\alpha} N_{\|}^{2} t^{2}\right) I_{\perp 1} H_{n \alpha}(t)
$$




$$
\begin{gathered}
+i \frac{k_{\perp}^{2}}{k^{2}} S_{n} \sin \psi \cos \psi\left[\frac { | n | } { T _ { n } ^ { - } } I _ { \perp 2 } ^ { - } \left(\chi_{n \alpha} t-\nu_{\alpha} \cos \psi\right.\right. \\
\left.\left.+i S_{n} \nu_{\alpha} \sin \psi\right)\left(\mu_{\alpha}^{1 / 2} \chi_{n \alpha} t\right)\right] \\
+i \frac{k_{\perp}^{2}}{k^{2}} S_{n} \sin \psi \cos \psi\left[\frac { | n | } { T _ { n } ^ { + } } I _ { \perp 2 } ^ { + } \left(-\chi_{n \alpha} t-\nu_{\alpha} \cos \psi\right.\right. \\
\left.\left.\quad-i S_{n} \nu_{\alpha} \sin \psi\right)\left(\mu_{\alpha}^{1 / 2} \chi_{n \alpha} t\right)\right] \\
+\frac{k_{\perp}^{2}}{k^{2}} \sin ^{2} \psi\left[\frac { | n | } { T _ { n } ^ { - } } I _ { \perp 2 } ^ { - } \left(-\chi_{n \alpha} t+\nu_{\alpha} \cos \psi\right.\right. \\
\left.\left.\quad-i S_{n} \nu_{\alpha} \sin \psi\right)\left(\mu_{\alpha}^{1 / 2} \chi_{n \alpha} t\right)\right] \\
+\frac{k_{\perp}^{2}}{k^{2}} \sin ^{2} \psi\left[\frac { | n | } { T _ { n } ^ { + } } I _ { \perp 2 } ^ { + } \left(-\chi_{n \alpha} t-\nu_{\alpha} \cos \psi\right.\right.
\end{gathered}
$$

$$
\begin{gathered}
\left.\left.-i S_{n} \nu_{\alpha} \sin \psi\right)\left(\mu_{\alpha}^{1 / 2} \chi_{n \alpha} t\right)\right] \\
-\frac{k_{\perp}^{2}}{k^{2}} \sin ^{2} \psi\left[\frac{H_{n \alpha}(t)}{S_{n \alpha}(t)} I_{\perp 3}\left(\mu_{\alpha} \chi_{n \alpha}^{2} t^{2}\right)\right] \\
+\frac{2 k_{\|} k_{\perp}}{k^{2}} S_{n} \cos \psi\left(-i N_{\|} t\right)|n| I_{\perp 1}\left(\mu_{\alpha}^{1 / 2} \nu_{\alpha} \cos \psi\right) \\
-i \frac{2 k_{\|} k_{\perp}}{k^{2}} \sin \psi\left(-i N_{\|} t\right) \mu_{\alpha}^{1 / 2}|n| I_{\perp 1}\left(\chi_{n \alpha} t+i S_{n} \nu_{\alpha} \sin \psi\right) \\
-i \frac{k_{\|} k_{\perp}}{k^{2}} \sin \psi\left(-i N_{\|} t\right) \mu_{\alpha}\left[\frac{H_{n \alpha}(t)}{T_{n}^{+}} I_{\perp 2}^{+} \chi_{n \alpha} t\right] \\
-i \frac{k_{\|} k_{\perp}}{k^{2}} \sin \psi\left(-i N_{\|} t\right) \mu_{\alpha}\left[\frac{H_{n \alpha}(t)}{T_{n}^{-}} I_{\perp 2}^{-} \chi_{n \alpha} t\right]
\end{gathered}
$$

Using the integrals given by Eqs. (21),

$$
\begin{aligned}
\varepsilon_{l}=1+ & i \sum_{\alpha} \frac{\omega_{\alpha}^{2}}{\omega^{2}} \mu_{\alpha}^{2} \sum_{n \rightarrow-\infty}^{\infty} \int_{0}^{\infty} d t \mathrm{e}^{i \mu_{\alpha}\left(1-n Y_{\alpha}\right) t} e^{-\mu_{\alpha} N_{\|}^{2} t^{2} / 2} \\
& \times \frac{\mathrm{e}^{-\left(\nu_{\alpha}^{2}+\chi_{n \alpha}^{2} t^{2}\right) /\left(1+i \zeta_{\alpha} t\right)}}{\mu_{\alpha}\left(1+i \zeta_{\alpha} t\right)} \frac{\left[H_{n \alpha}(t)\right]^{(|n|-1)}}{\left(S_{n \alpha}(t)\right)^{|n|}} \Lambda^{*}
\end{aligned}
$$

where

$$
\begin{gathered}
\Lambda^{*}=\frac{k_{\perp}^{2}}{k^{2}} n^{2} I_{|n|}\left(\frac{S_{n \alpha}(t)}{1+i \zeta_{\alpha} t}\right) \\
+\frac{k_{\|}^{2}}{k^{2}}\left(1-\mu_{\alpha} N_{||}^{2} t^{2}\right) I_{|n|}\left(\frac{S_{n \alpha}(t)}{1+i \zeta_{\alpha} t}\right) H_{n \alpha}(t) \\
+i \frac{k_{\perp}^{2}}{k^{2}} S_{n} \frac{\sin \psi \cos \psi}{\left(1+i \zeta_{\alpha} t\right)} \\
\times\left[\frac{|n|}{T_{n}^{-}}\left[T_{n}^{-} I_{|n|}\left(\frac{S_{n \alpha}(t)}{1+i \zeta_{\alpha} t}\right)-T_{n}^{+} I_{|n|+1}\left(\frac{S_{n \alpha}(t)}{1+i \zeta_{\alpha} t}\right)\right]\right. \\
\left.\times\left(\chi_{n \alpha} t-\nu_{\alpha} \cos \psi+i S_{n} \nu_{\alpha} \sin \psi\right)\left(\chi_{n \alpha} t\right)\right] \\
+i \frac{k_{\perp}^{2}}{k^{2}} S_{n} \frac{\sin \psi \cos \psi}{\left(1+i \zeta_{\alpha} t\right)} \\
\times\left[\frac{|n|}{T_{n}^{+}}\left[T_{n}^{+} I_{|n|}\left(\frac{S_{n \alpha}(t)}{1+i \zeta_{\alpha} t}\right)-T_{n}^{-} I_{|n|+1}\left(\frac{S_{n \alpha}(t)}{1+i \zeta_{\alpha} t}\right)\right]\right. \\
\left.\times\left(-\chi_{n \alpha} t-\nu_{\alpha} \cos \psi-i S_{n} \nu_{\alpha} \sin \psi\right)\left(\chi_{n \alpha} t\right)\right] \\
+\frac{k_{\perp}^{2}}{k^{2}} \frac{\sin ^{2} \psi}{\left(1+i \zeta_{\alpha} t\right)} \\
\times\left[\frac{|n|}{T_{n}^{+}}\left[T_{n}^{+} I_{|n|}\left(\frac{S_{n \alpha}(t)}{1+i \zeta_{\alpha} t}\right)-T_{n}^{-} I_{|n|+1}\left(\frac{\sin ^{2} \psi}{1+i \zeta_{\alpha} t}\right)\right]\right. \\
\times\left(-\chi_{n \alpha}^{-} I_{|n|}\left(\frac{S_{n \alpha}(t)}{1+i \zeta_{\alpha} t}\right)-T_{n}^{+} I_{|n|+1}\left(\frac{S_{n \alpha}(t)}{1+i \zeta_{\alpha} t}\right)\right] \\
\left.\times\left(-\chi_{n \alpha} t+\nu_{\alpha} \cos \psi-i S_{n} \nu_{\alpha} \sin \psi\right)\left(\chi_{n \alpha} t\right)\right] \\
\times
\end{gathered}
$$




$$
\begin{gathered}
-\frac{k_{\perp}^{2}}{k^{2}} \frac{2 \sin ^{2} \psi}{\left(1+i \zeta_{\alpha} t\right)} \\
\times\left[\frac{H_{n \alpha}(t)}{S_{n \alpha}(t)}\left(\chi_{n \alpha}^{2} t^{2}\right)\right]\left[\frac{S_{n \alpha}(t)}{1+i \zeta_{\alpha} t} I_{|n|}\left(\frac{S_{n \alpha}(t)}{1+i \zeta_{\alpha} t}\right)\right. \\
\left.-\left(|n|+\frac{\nu_{\alpha}^{2}+\chi_{n \alpha}^{2} t^{2}}{1+i \zeta_{\alpha} t}\right) I_{|n|+1}\left(\frac{S_{n \alpha}(t)}{1+i \zeta_{\alpha} t}\right)\right] \\
-\frac{2 k_{\|} k_{\perp}}{k^{2}}\left(N_{||} t\right)|n| \mu_{\alpha}^{1 / 2}\left[i S_{n} \nu_{\alpha}+\sin \psi \chi_{n \alpha} t\right] I_{|n|}\left(\frac{S_{n \alpha}(t)}{1+i \zeta_{\alpha} t}\right) \\
\times\left[\frac{H_{n \alpha}(t)}{T_{n}^{+}}\left[T_{n}^{+} I_{|n|}\left(\frac{S_{n \alpha}(t)}{1+i \zeta_{\alpha} t}\right)-T_{n}^{-} I_{|n|+1}\left(\frac{S_{n \alpha}(t)}{1+i \zeta_{\alpha} t}\right)\right] \chi_{n \alpha} t\right] \\
-i \frac{k_{\|} k_{\perp}}{k^{2}} \frac{\sin \psi\left(-i N_{\| \mid} t\right)}{\left(1+i \zeta_{\alpha} t\right)} \mu_{\alpha}^{1 / 2} \mu_{\alpha}^{1 / 2} \\
\times\left[\frac{H_{n \alpha}(t)}{T_{n}^{-}}\left[T_{n}^{-} I_{|n|}\left(\frac{S_{n \alpha}(t)}{1+i \zeta_{\alpha} t}\right)-T_{n}^{+} I_{|n|+1}\left(\frac{S_{n \alpha}(t)}{1+i \zeta_{\alpha} t}\right)\right] \chi_{n \alpha} t\right]
\end{gathered}
$$

where we have taken into account the following

$$
\begin{gathered}
\frac{2 k_{\|} k_{\perp}}{k^{2}} S_{n} \cos \psi\left(-i N_{\|} t\right)|n|\left(\mu_{\alpha}^{1 / 2} \nu_{\alpha} \cos \psi\right) I_{|n|}\left(\frac{S_{n \alpha}(t)}{1+i \zeta_{\alpha} t}\right) \\
-\frac{2 k_{\|} k_{\perp}}{k^{2}} \sin \psi\left(N_{\|} t\right)|n| \\
\times\left(\mu_{\alpha}^{1 / 2} \chi_{n \alpha} t+i S_{n} \mu_{\alpha}^{1 / 2} \nu_{\alpha} \sin \psi\right) I_{|n|}\left(\frac{S_{n \alpha}(t)}{1+i \zeta_{\alpha} t}\right) \\
=\frac{2 k_{\|} k_{\perp}}{k^{2}}\left(N_{\|} t\right)|n| \mu_{\alpha}^{1 / 2}\left[-i S_{n} \nu_{\alpha}\left(\cos ^{2} \psi+\sin ^{2} \psi\right)\right. \\
\left.-\sin \psi \chi_{n \alpha} t\right] I_{|n|}\left(\frac{S_{n \alpha}(t)}{1+i \zeta_{\alpha} t}\right) \\
=-\frac{2 k_{\|} k_{\perp}}{k^{2}}\left(N_{\|} t\right)|n| \mu_{\alpha}^{1 / 2}\left[i S_{n} \nu_{\alpha}+\sin \psi \chi_{n \alpha} t\right] \\
\times I_{|n|}\left(\frac{S_{n \alpha}(t)}{1+i \zeta_{\alpha} t}\right) .
\end{gathered}
$$

We now introduce the definition of the "inhomogeneous plasma dispersion function", for the non-relativistic case [5]

$$
\begin{gathered}
\mathcal{G}_{r, p, m, l}=-i \int_{0}^{\infty} d t \frac{(i t)^{r} \mathrm{e}^{i \mu_{\alpha}\left(1-n Y_{\alpha}\right) t} e^{-\mu_{\alpha} N_{\|}^{2} t^{2} / 2}}{\left(1+i \zeta_{\alpha} t\right)^{p}} \\
\times \mathrm{e}^{-\left(\nu_{\alpha}^{2}+\chi_{n \alpha}^{2} t^{2}\right) /\left(1+i \zeta_{\alpha} t\right)} \frac{\left[H_{n \alpha}(t)\right]^{m}}{\left(S_{n \alpha}(t)\right)^{l}} I_{l}\left(\frac{S_{n \alpha}(t)}{1+i \zeta_{\alpha} t}\right)
\end{gathered}
$$

Eq. (26) can be prepared for the use of the inhomogeneous plasma dispersion function, as follows,

$$
\begin{gathered}
\Lambda^{*}=\frac{k_{\perp}^{2}}{k^{2}} n^{2} I_{|n|}\left(\frac{S_{n \alpha}(t)}{1+i \zeta_{\alpha} t}\right) \\
+\frac{k_{\|}^{2}}{k^{2}}\left(1+\mu_{\alpha} N_{\|}^{2}(i t)^{2}\right) I_{|n|}\left(\frac{S_{n \alpha}(t)}{1+i \zeta_{\alpha} t}\right) H_{n \alpha}(t) \\
+\frac{k_{\perp}^{2}}{k^{2}} S_{n}|n| \chi_{n \alpha} \frac{\sin \psi \cos \psi}{\left(1+i \zeta_{\alpha} t\right)} I_{|n|}\left(\frac{S_{n \alpha}(t)}{1+i \zeta_{\alpha} t}\right)
\end{gathered}
$$




$$
\begin{aligned}
& \times\left(-i \chi_{n \alpha} i t-\nu_{\alpha} \cos \psi+i S_{n} \nu_{\alpha} \sin \psi\right)(i t) \\
& -\frac{k_{\perp}^{2}}{k^{2}} S_{n}|n| \chi_{n \alpha} \frac{\sin \psi \cos \psi}{\left(1+i \zeta_{\alpha} t\right)} \frac{T_{n}^{+}}{T_{n}^{-}} I_{|n|+1}\left(\frac{S_{n \alpha}(t)}{1+i \zeta_{\alpha} t}\right) \\
& \times\left(-i \chi_{n \alpha} i t-\nu_{\alpha} \cos \psi+i S_{n} \nu_{\alpha} \sin \psi\right)(i t) \\
& +\frac{k_{\perp}^{2}}{k^{2}} S_{n}|n| \chi_{n \alpha} \frac{\sin \psi \cos \psi}{\left(1+i \zeta_{\alpha} t\right)} I_{|n|}\left(\frac{S_{n \alpha}(t)}{1+i \zeta_{\alpha} t}\right) \\
& \times\left(i \chi_{n \alpha} i t-\nu_{\alpha} \cos \psi-i S_{n} \nu_{\alpha} \sin \psi\right)(i t) \\
& -\frac{k_{\perp}^{2}}{k^{2}} S_{n}|n| \chi_{n \alpha} \frac{\sin \psi \cos \psi}{\left(1+i \zeta_{\alpha} t\right)} \frac{T_{n}^{-}}{T_{n}^{+}} I_{|n|+1}\left(\frac{S_{n \alpha}(t)}{1+i \zeta_{\alpha} t}\right) \\
& \times\left(i \chi_{n \alpha} i t-\nu_{\alpha} \cos \psi-i S_{n} \nu_{\alpha} \sin \psi\right)(i t) \\
& -i \frac{k_{\perp}^{2}}{k^{2}}|n| \chi_{n \alpha} \frac{\sin ^{2} \psi}{\left(1+i \zeta_{\alpha} t\right)} I_{|n|}\left(\frac{S_{n \alpha}(t)}{1+i \zeta_{\alpha} t}\right) \\
& \times\left(i \chi_{n \alpha} i t+\nu_{\alpha} \cos \psi-i S_{n} \nu_{\alpha} \sin \psi\right)(i t) \\
& +i \frac{k_{\perp}^{2}}{k^{2}}|n| \chi_{n \alpha} \frac{\sin ^{2} \psi}{\left(1+i \zeta_{\alpha} t\right)} \frac{T_{n}^{+}}{T_{n}^{-}} I_{|n|+1}\left(\frac{S_{n \alpha}(t)}{1+i \zeta_{\alpha} t}\right) \\
& \times\left(i \chi_{n \alpha} i t+\nu_{\alpha} \cos \psi-i S_{n} \nu_{\alpha} \sin \psi\right)(i t) \\
& -i \frac{k_{\perp}^{2}}{k^{2}}|n| \chi_{n \alpha} \frac{\sin ^{2} \psi}{\left(1+i \zeta_{\alpha} t\right)} I_{|n|}\left(\frac{S_{n \alpha}(t)}{1+i \zeta_{\alpha} t}\right) \\
& \times\left(i \chi_{n \alpha} i t-\nu_{\alpha} \cos \psi-i S_{n} \nu_{\alpha} \sin \psi\right)(i t) \\
& +i \frac{k_{\perp}^{2}}{k^{2}}|n| \chi_{n \alpha} \frac{\sin ^{2} \psi}{\left(1+i \zeta_{\alpha} t\right)} \frac{T_{n}^{-}}{T_{n}^{+}} I_{|n|+1}\left(\frac{S_{n \alpha}(t)}{1+i \zeta_{\alpha} t}\right) \\
& \times\left(i \chi_{n \alpha} i t-\nu_{\alpha} \cos \psi-i S_{n} \nu_{\alpha} \sin \psi\right)(i t) \\
& +\frac{k_{\perp}^{2}}{k^{2}} \chi_{n \alpha}^{2} \frac{2 \sin ^{2} \psi}{\left(1+i \zeta_{\alpha} t\right)}\left[\frac{H_{n \alpha}(t)}{S_{n \alpha}(t)}(i t)^{2}\right]\left[\frac{S_{n \alpha}(t)}{1+i \zeta_{\alpha} t} I_{|n|}\left(\frac{S_{n \alpha}(t)}{1+i \zeta_{\alpha} t}\right)\right. \\
& \left.-\left(|n|+\frac{\nu_{\alpha}^{2}-\chi_{n \alpha}^{2}(i t)^{2}}{1+i \zeta_{\alpha} t}\right) I_{|n|+1}\left(\frac{S_{n \alpha}(t)}{1+i \zeta_{\alpha} t}\right)\right] \\
& -\frac{2 k_{\|} k_{\perp}}{k^{2}} N_{\|}(i t)|n| \mu_{\alpha}^{1 / 2}\left[S_{n} \nu_{\alpha}-\sin \psi \chi_{n \alpha}(i t)\right] I_{|n|}\left(\frac{S_{n \alpha}(t)}{1+i \zeta_{\alpha} t}\right) \\
& +\frac{k_{\|} k_{\perp}}{k^{2}} N_{\|} \chi_{n \alpha} \mu_{\alpha}^{1 / 2} \frac{\sin \psi}{\left(1+i \zeta_{\alpha} t\right)} H_{n \alpha}(t) I_{|n|}\left(\frac{S_{n \alpha}(t)}{1+i \zeta_{\alpha} t}\right)(i t)^{2} \\
& -\frac{k_{\|} k_{\perp}}{k^{2}} N_{\|} \chi_{n \alpha} \mu_{\alpha}^{1 / 2} \frac{\sin \psi}{\left(1+i \zeta_{\alpha} t\right)} \frac{T_{n}^{-}}{T_{n}^{+}} H_{n \alpha}(t) I_{|n|+1}\left(\frac{S_{n \alpha}(t)}{1+i \zeta_{\alpha} t}\right)(i t)^{2} \\
& +\frac{k_{\|} k_{\perp}}{k^{2}} N_{\|} \chi_{n \alpha} \mu_{\alpha}^{1 / 2} \frac{\sin \psi}{\left(1+i \zeta_{\alpha} t\right)} H_{n \alpha}(t) I_{|n|}\left(\frac{S_{n \alpha}(t)}{1+i \zeta_{\alpha} t}\right)(i t)^{2} \\
& -\frac{k_{\|} k_{\perp}}{k^{2}} N_{\|} \chi_{n \alpha} \mu_{\alpha}^{1 / 2} \frac{\sin \psi}{\left(1+i \zeta_{\alpha} t\right)} \frac{T_{n}^{+}}{T_{n}^{-}} H_{n \alpha}(t) I_{|n|+1}\left(\frac{S_{n \alpha}(t)}{1+i \zeta_{\alpha} t}\right)(i t)^{2}
\end{aligned}
$$

Using the definition of the inhomogeneous dispersion function, we obtain the following

$$
\begin{aligned}
\varepsilon_{l}= & -\sum_{\alpha} \frac{\omega_{\alpha}^{2}}{\omega^{2}} \mu_{\alpha} \sum_{n \rightarrow-\infty}^{\infty}\left\{\frac{k_{\perp}^{2}}{k^{2}} n^{2} \mathcal{G}_{0,1,|n|-1,|n|}\right. \\
& +\frac{k_{\|}^{2}}{k^{2}}\left(\mathcal{G}_{0,1,|n|,|n|}+\mu_{\alpha} N_{\|}^{2} \mathcal{G}_{2,1,|n|,|n|}\right) \\
+ & \frac{k_{\perp}^{2}}{k^{2}} S_{n}|n| \chi_{n \alpha} \sin \psi \cos \psi\left(-i \chi_{n \alpha} \mathcal{G}_{2,2,|n|-1,|n|}\right.
\end{aligned}
$$




$$
\begin{aligned}
& \left.-\nu_{\alpha} \cos \psi \mathcal{G}_{1,2,|n|-1,|n|}+i S_{n} \nu_{\alpha} \sin \psi \mathcal{G}_{1,2,|n|-1,|n|}\right) \\
& +\frac{k_{\perp}^{2}}{k^{2}} S_{n}|n| \chi_{n \alpha} \sin \psi \cos \psi\left(i \chi_{n \alpha} \mathcal{G}_{2,2,|n|-1,|n|}\right. \\
& \left.-\nu_{\alpha} \cos \psi \mathcal{G}_{1,2,|n|-1,|n|}-i S_{n} \nu_{\alpha} \sin \psi \mathcal{G}_{1,2,|n|-1,|n|}\right) \\
& -i \frac{k_{\perp}^{2}}{k^{2}}|n| \chi_{n \alpha} \sin ^{2} \psi\left(i \chi_{n \alpha} \mathcal{G}_{2,2,|n|-1,|n|}\right. \\
& \left.+\nu_{\alpha} \cos \psi \mathcal{G}_{1,2,|n|-1,|n|}-i S_{n} \nu_{\alpha} \sin \psi \mathcal{G}_{1,2,|n|-1,|n|}\right) \\
& -i \frac{k_{\perp}^{2}}{k^{2}}|n| \chi_{n \alpha} \sin ^{2} \psi\left(i \chi_{n \alpha} \mathcal{G}_{2,2,|n|-1,|n|}\right. \\
& \left.-\nu_{\alpha} \cos \psi \mathcal{G}_{1,2,|n|-1,|n|}-i S_{n} \nu_{\alpha} \sin \psi \mathcal{G}_{1,2,|n|-1,|n|}\right) \\
& +2 \frac{k_{\perp}^{2}}{k^{2}} \chi_{n \alpha}^{2} \sin ^{2} \psi\left[\mathcal{G}_{2,3,|n|,|n|}-|n| \mathcal{G}_{2,2,|n|,|n|+1}\right. \\
& \left.-\nu_{\alpha}^{2} \mathcal{G}_{2,3,|n|,|n|+1}+\chi_{n \alpha}^{2} \mathcal{G}_{4,3,|n|,|n|+1}\right] \\
& -\frac{2 k_{\|} k_{\perp}}{k^{2}} N_{\|}|n| \mu_{\alpha}^{1 / 2}\left[S_{n} \nu_{\alpha} \mathcal{G}_{1,1,|n|-1,|n|}-\sin \psi \chi_{n \alpha} \mathcal{G}_{2,1,|n|-1,|n|}\right] \\
& +\frac{k_{\|} k_{\perp}}{k^{2}} N_{\|} \chi_{n \alpha} \mu_{\alpha}^{1 / 2} \sin \psi \mathcal{G}_{2,2,|n|,|n|} \\
& \left.+\frac{k_{\|} k_{\perp}}{k^{2}} N_{\|} \chi_{n \alpha} \mu_{\alpha}^{1 / 2} \sin \psi \mathcal{G}_{2,2,|n|,|n|}\right\} \\
& +i \sum_{\alpha} \frac{\omega_{\alpha}^{2}}{\omega^{2}} \mu_{\alpha}^{2} \sum_{n \rightarrow-\infty}^{\infty} \int_{0}^{\infty} d t \mathrm{e}^{i \mu_{\alpha}\left(1-n Y_{\alpha}\right) t} e^{-\mu_{\alpha} N_{\|}^{2} t^{2} / 2} \\
& \times \frac{\mathrm{e}^{-\left(\nu_{\alpha}^{2}+\chi_{n \alpha}^{2} t^{2}\right) /\left(1+i \zeta_{\alpha} t\right)}}{\mu_{\alpha}\left(1+i \zeta_{\alpha} t\right)} \frac{\left[H_{n \alpha}(t)\right]^{(|n|-1)}}{\left(S_{n \alpha}(t)\right)^{|n|}} \Lambda^{\dagger}
\end{aligned}
$$

where

$$
\begin{aligned}
& \Lambda^{\dagger}=-\frac{k_{\perp}^{2}}{k^{2}} S_{n}|n| \chi_{n \alpha} \frac{\sin \psi \cos \psi}{\left(1+i \zeta_{\alpha} t\right)} \frac{T_{n}^{+}}{T_{n}^{-}} I_{|n|+1}\left(\frac{S_{n \alpha}(t)}{1+i \zeta_{\alpha} t}\right) \\
& \times\left(-i \chi_{n \alpha} i t-\nu_{\alpha} \cos \psi+i S_{n} \nu_{\alpha} \sin \psi\right)(i t) \\
& -\frac{k_{\perp}^{2}}{k^{2}} S_{n}|n| \chi_{n \alpha} \frac{\sin \psi \cos \psi}{\left(1+i \zeta_{\alpha} t\right)} \frac{T_{n}^{-}}{T_{n}^{+}} I_{|n|+1}\left(\frac{S_{n \alpha}(t)}{1+i \zeta_{\alpha} t}\right) \\
& \times\left(i \chi_{n \alpha} i t-\nu_{\alpha} \cos \psi-i S_{n} \nu_{\alpha} \sin \psi\right)(i t) \\
& +i \frac{k_{\perp}^{2}}{k^{2}}|n| \chi_{n \alpha} \frac{\sin ^{2} \psi}{\left(1+i \zeta_{\alpha} t\right)} \frac{T_{n}^{+}}{T_{n}^{-}} I_{|n|+1}\left(\frac{S_{n \alpha}(t)}{1+i \zeta_{\alpha} t}\right) \\
& \times\left(i \chi_{n \alpha} i t+\nu_{\alpha} \cos \psi-i S_{n} \nu_{\alpha} \sin \psi\right)(i t) \\
& +i \frac{k_{\perp}^{2}}{k^{2}}|n| \chi_{n \alpha} \frac{\sin ^{2} \psi}{\left(1+i \zeta_{\alpha} t\right)} \frac{T_{n}^{-}}{T_{n}^{+}} I_{|n|+1}\left(\frac{S_{n \alpha}(t)}{1+i \zeta_{\alpha} t}\right) \\
& \times\left(i \chi_{n \alpha} i t-\nu_{\alpha} \cos \psi-i S_{n} \nu_{\alpha} \sin \psi\right)(i t) \\
& -\frac{k_{\|} k_{\perp}}{k^{2}} N_{\|} \chi_{n \alpha} \mu_{\alpha}^{1 / 2} \frac{\sin \psi}{\left(1+i \zeta_{\alpha} t\right)} \frac{T_{n}^{-}}{T_{n}^{+}} H_{n \alpha}(t) \\
& \times I_{|n|+1}\left(\frac{S_{n \alpha}(t)}{1+i \zeta_{\alpha} t}\right)(i t)^{2} \\
& -\frac{k_{\|} k_{\perp}}{k^{2}} N_{\|} \chi_{n \alpha} \mu_{\alpha}^{1 / 2} \frac{\sin \psi}{\left(1+i \zeta_{\alpha} t\right)} \frac{T_{n}^{+}}{T_{n}^{-}} H_{n \alpha}(t) \\
& \times I_{|n|+1}\left(\frac{S_{n \alpha}(t)}{1+i \zeta_{\alpha} t}\right)(i t)^{2} .
\end{aligned}
$$


This expression can be somewhat simplified, by collecting together some terms and by cancelling others

$$
\begin{aligned}
& \varepsilon_{l}=1-\sum_{\alpha} \frac{\omega_{\alpha}^{2}}{\omega^{2}} \mu_{\alpha} \sum_{n \rightarrow-\infty}^{\infty}\left\{\frac{k_{\perp}^{2}}{k^{2}} n^{2} \mathcal{G}_{0,1,|n|-1,|n|}\right. \\
& +\frac{k_{\|}^{2}}{k^{2}}\left(\mathcal{G}_{0,1,|n|,|n|}+\mu_{\alpha} N_{\|}^{2} \mathcal{G}_{2,1,|n|,|n|}\right) \\
& -2 \frac{k_{\perp}^{2}}{k^{2}} n \nu_{\alpha} \chi_{n \alpha} \sin \psi \mathcal{G}_{1,2,|n|-1,|n|} \\
& +2 \frac{k_{\perp}^{2}}{k^{2}} \chi_{n \alpha}^{2} \sin ^{2} \psi\left[\mathcal{G}_{2,3,|n|,|n|}+|n| \mathcal{G}_{2,2,|n|-1,|n|}\right. \\
& \left.-|n| \mathcal{G}_{2,2,|n|,|n|+1}-\nu_{\alpha}^{2} \mathcal{G}_{2,3,|n|,|n|+1}+\chi_{n \alpha}^{2} \mathcal{G}_{4,3,|n|,|n|+1}\right] \\
& -\frac{2 k_{\|} k_{\perp}}{k^{2}} N_{\|}|n| \mu_{\alpha}^{1 / 2}\left[S_{n} \nu_{\alpha} \mathcal{G}_{1,1,|n|-1,|n|}-\sin \psi \chi_{n \alpha} \mathcal{G}_{2,1,|n|-1,|n|}\right] \\
& \left.+2 \frac{k_{\|} k_{\perp}}{k^{2}} N_{\|} \chi_{n \alpha} \mu_{\alpha}^{1 / 2} \sin \psi \mathcal{G}_{2,2,|n|,|n|}\right\} \\
& +i \sum_{\alpha} \frac{\omega_{\alpha}^{2}}{\omega^{2}} \mu_{\alpha}^{2} \sum_{n \rightarrow-\infty}^{\infty} \int_{0}^{\infty} d t \mathrm{e}^{i \mu_{\alpha}\left(1-n Y_{\alpha}\right) t} e^{-\mu_{\alpha} N_{\|}^{2} t^{2} / 2} \\
& \times \frac{\mathrm{e}^{-\left(\nu_{\alpha}^{2}+\chi_{n \alpha}^{2} t^{2}\right) /\left(1+i \zeta_{\alpha} t\right)}}{\mu_{\alpha}\left(1+i \zeta_{\alpha} t\right)} \frac{\left[H_{n \alpha}(t)\right]^{(|n|-1)}}{\left(S_{n \alpha}(t)\right)^{|n|}} \Lambda^{\dagger} .
\end{aligned}
$$

Let us examine the terms appearing in the expression for $\Lambda^{\dagger}$. Initially we consider the following terms,

$$
\begin{aligned}
&-\frac{k_{\perp}^{2}}{k^{2}} S_{n}|n| \frac{\sin \psi \cos \psi}{\left(1+i \zeta_{\alpha} t\right)} \frac{T_{n}^{+}}{T_{n}^{-}} I_{|n|+1}\left(\frac{S_{n \alpha}(t)}{1+i \zeta_{\alpha} t}\right) \\
& \times\left(-i \chi_{n \alpha} i t-\nu_{\alpha} \cos \psi+i S_{n} \nu_{\alpha} \sin \psi\right)\left(\chi_{n \alpha} i t\right) \\
&- \frac{k_{\perp}^{2}}{k^{2}} S_{n}|n| \frac{\sin \psi \cos \psi}{\left(1+i \zeta_{\alpha} t\right)} \frac{T_{n}^{-}}{T_{n}^{+}} I_{|n|+1}\left(\frac{S_{n \alpha}(t)}{1+i \zeta_{\alpha} t}\right) \\
& \times\left(i \chi_{n \alpha} i t-\nu_{\alpha} \cos \psi-i S_{n} \nu_{\alpha} \sin \psi\right)\left(\chi_{n \alpha} i t\right) \\
&= \frac{k_{\perp}^{2}}{k^{2}} S_{n}|n| \frac{\sin \psi \cos \psi}{\left(1+i \zeta_{\alpha} t\right)} I_{|n|+1}\left(\frac{S_{n \alpha}(t)}{1+i \zeta_{\alpha} t}\right)\left(\chi_{n \alpha} i t\right) \\
& \times {\left[-i \chi_{n \alpha} i t\left(\frac{T_{n}^{+}}{T_{n}^{-}}-\frac{T_{n}^{-}}{T_{n}^{+}}\right)\right.} \\
&\left.-\nu_{\alpha} \cos \psi\left(\frac{T_{n}^{+}}{T_{n}^{-}}+\frac{T_{n}^{-}}{T_{n}^{+}}\right)+i S_{n} \nu_{\alpha} \sin \psi\left(\frac{T_{n}^{+}}{T_{n}^{-}}-\frac{T_{n}^{-}}{T_{n}^{+}}\right)\right] .
\end{aligned}
$$

Now we may consider the following,

$$
\begin{aligned}
\frac{T_{n}^{-}}{T_{n}^{+}}= & \frac{T_{n}^{-} T_{n}^{+}}{T_{n}^{+} T_{n}^{+}}=\frac{\left(\sqrt{\nu_{\alpha}^{2}+2 \nu_{\alpha} \cos \psi \chi_{n \alpha} t+\chi_{n \alpha}^{2} t^{2}}\right)}{\left(\sqrt{\nu_{\alpha}^{2}+2 \nu_{\alpha} \cos \psi \chi_{n \alpha} t+\chi_{n \alpha}^{2} t^{2}}\right)} \\
& \times \frac{\left(\sqrt{\nu_{\alpha}^{2}-2 \nu_{\alpha} \cos \psi \chi_{n \alpha} t+\chi_{n \alpha}^{2} t^{2}}\right)}{\left(\sqrt{\nu_{\alpha}^{2}+2 \nu_{\alpha} \cos \psi \chi_{n \alpha} t+\chi_{n \alpha}^{2} t^{2}}\right)} \\
= & \frac{\sqrt{\nu_{\alpha}^{4}+\chi_{n \alpha}^{4} t^{4}-2 \nu_{\alpha}^{2} \cos (2 \psi) \chi_{n \alpha}^{2} t^{2}}}{\nu_{\alpha}^{2}+2 \nu_{\alpha} \cos \psi \chi_{n \alpha} t+\chi_{n \alpha}^{2} t^{2}} \\
& =\frac{S_{n \alpha}(t)}{\left|\nu_{\alpha}^{2}+2 \nu_{\alpha} \cos \psi \chi_{n \alpha} t+\chi_{n \alpha}^{2} t^{2}\right|}
\end{aligned}
$$


This expression can be inverted, and we obtain,

$$
\frac{T_{n}^{+}}{T_{n}^{-}}=\frac{\nu_{\alpha}^{2}+2 \nu_{\alpha} \cos \psi \chi_{n \alpha} t+\chi_{n \alpha}^{2} t^{2}}{S_{n \alpha}(t)}
$$

Multiplying numerator and denominator by $T_{n}^{-}$,

$$
\begin{gathered}
\frac{T_{n}^{-}}{T_{n}^{+}}=\frac{T_{n}^{-} T_{n}^{-}}{T_{n}^{+} T_{n}^{-}}=\frac{\left(\sqrt{\nu_{\alpha}^{2}-2 \nu_{\alpha} \cos \psi \chi_{n \alpha} t+\chi_{n \alpha}^{2} t^{2}}\right)}{\left(\sqrt{\nu_{\alpha}^{2}-2 \nu_{\alpha} \cos \psi \chi_{n \alpha} t+\chi_{n \alpha}^{2} t^{2}}\right)} \\
\quad \times \frac{\left(\sqrt{\nu_{\alpha}^{2}-2 \nu_{\alpha} \cos \psi \chi_{n \alpha} t+\chi_{n \alpha}^{2} t^{2}}\right)}{\left(\sqrt{\nu_{\alpha}^{2}+2 \nu_{\alpha} \cos \psi \chi_{n \alpha} t+\chi_{n \alpha}^{2} t^{2}}\right)} \\
=\frac{\nu_{\alpha}^{2}-2 \nu_{\alpha} \cos \psi \chi_{n \alpha} t+\chi_{n \alpha}^{2} t^{2}}{\sqrt{\nu_{\alpha}^{4}+\chi_{n \alpha}^{4} t^{4}-2 \nu_{\alpha}^{2} \cos (2 \psi) \chi_{n \alpha}^{2} t^{2}}} \\
=\frac{\nu_{\alpha}^{2}-2 \nu_{\alpha} \cos \psi \chi_{n \alpha} t+\chi_{n \alpha}^{2} t^{2}}{S_{n \alpha}(t)}
\end{gathered}
$$

Consider now Eq. (30),

$$
\begin{gathered}
\frac{T_{n}^{+}}{T_{n}^{-}}=\frac{\nu_{\alpha}^{2}+2 \nu_{\alpha} \cos \psi \chi_{n \alpha} t+\chi_{n \alpha}^{2} t^{2}}{S_{n \alpha}(t)} \\
=\frac{H_{n \alpha}(t)}{S_{n \alpha}(t)}+\frac{2 \nu_{\alpha} \chi_{n \alpha} t}{S_{n \alpha}(t)}\left(\cos \psi+i S_{n} \sin \psi\right)+\frac{2 \chi_{n \alpha}^{2} t^{2}}{S_{n \alpha}(t)} .
\end{gathered}
$$

Taking into account the following property,

$$
e^{ \pm i S_{n} \psi}=\cos \left(S_{n} \psi\right) \pm i \sin \left(S_{n} \psi\right)=\cos (\psi) \pm i S_{n} \sin (\psi)
$$

we obtain,

$$
\frac{T_{n}^{+}}{T_{n}^{-}}=\frac{H_{n \alpha}(t)}{S_{n \alpha}(t)}+\frac{2 \nu_{\alpha} \chi_{n \alpha} t}{S_{n \alpha}(t)} e^{i S_{n} \psi}+\frac{2 \chi_{n \alpha}^{2} t^{2}}{S_{n \alpha}(t)} .
$$

In analogous way,

$$
\begin{gathered}
\frac{T_{n}^{-}}{T_{n}^{+}}=\frac{\nu_{\alpha}^{2}-2 \nu_{\alpha} \cos \psi \chi_{n \alpha} t+\chi_{n \alpha}^{2} t^{2}}{S_{n \alpha}(t)} \\
=\frac{H_{n \alpha}(t)}{S_{n \alpha}(t)}-\frac{2 \nu_{\alpha} \chi_{n \alpha} t}{S_{n \alpha}(t)}\left(\cos \psi-i S_{n} \sin \psi\right)+\frac{2 \chi_{n \alpha}^{2} t^{2}}{S_{n \alpha}(t)} \\
=\frac{H_{n \alpha}(t)}{S_{n \alpha}(t)}-\frac{2 \nu_{\alpha} \chi_{n \alpha} t}{S_{n \alpha}(t)} e^{-i S_{n} \psi}+\frac{2 \chi_{n \alpha}^{2} t^{2}}{S_{n \alpha}(t)} .
\end{gathered}
$$

Collecting together these results,

$$
\frac{T_{n}^{ \pm}}{T_{n}^{\mp}}=\frac{H_{n \alpha}(t)}{S_{n \alpha}(t)} \pm \frac{2 \nu_{\alpha} \chi_{n \alpha} t}{S_{n \alpha}(t)} e^{ \pm i S_{n} \psi}+\frac{2 \chi_{n \alpha}^{2} t^{2}}{S_{n \alpha}(t)} .
$$

Therefore, we have,

$$
\begin{gathered}
\left(\frac{T_{n}^{+}}{T_{n}^{-}}-\frac{T_{n}^{-}}{T_{n}^{+}}\right)=\frac{2 \nu_{\alpha} \chi_{n \alpha} t}{S_{n \alpha}(t)}\left(e^{i S_{n} \psi}+e^{-i S_{n} \psi}\right) \\
=-i \frac{4 \nu_{\alpha} \chi_{n \alpha}(i t)}{S_{n \alpha}(t)} \cos \psi \\
\left(\frac{T_{n}^{+}}{T_{n}^{-}}+\frac{T_{n}^{-}}{T_{n}^{+}}\right)=2 \frac{H_{n \alpha}(t)}{S_{n \alpha}(t)}+\frac{4 \chi_{n \alpha}^{2}(t)^{2}}{S_{n \alpha}(t)} \\
+\frac{2 \nu_{\alpha} \chi_{n \alpha} t}{S_{n \alpha}(t)}\left(e^{i S_{n} \psi}-e^{-i S_{n} \psi}\right)
\end{gathered}
$$




$$
\begin{gathered}
=\frac{2}{S_{n \alpha}(t)}\left[H_{n \alpha}(t)+2 \chi_{n \alpha}^{2}(t)^{2}+i S_{n} 2 \nu_{\alpha} \chi_{n \alpha} t \sin \psi\right] \\
=\frac{2}{S_{n \alpha}(t)}\left[\nu_{\alpha}^{2}+\chi_{n \alpha}^{2} t^{2}\right] .
\end{gathered}
$$

Using these results, a small amount of algebra transforms Eq. (29) into the following

$$
2 \frac{k_{\perp}^{2}}{k^{2}} n \frac{\sin \psi \cos ^{2} \psi}{\left(1+i \zeta_{\alpha} t\right)} I_{|n|+1}\left(\frac{S_{n \alpha}(t)}{1+i \zeta_{\alpha} t}\right)\left(\nu_{\alpha} \chi_{n \alpha} i t\right) \frac{H_{n \alpha}(t)}{S_{n \alpha}(t)} .
$$

The next two terms in the expression for $\Lambda^{\dagger}$ are the following

$$
\begin{aligned}
& i \frac{k_{\perp}^{2}}{k^{2}} \frac{\sin ^{2} \psi}{\left(1+i \zeta_{\alpha} t\right)}|n| \frac{T_{n}^{+}}{T_{n}^{-}} I_{|n|+1}\left(\frac{S_{n \alpha}(t)}{1+i \zeta_{\alpha} t}\right) \\
\times & \left(i \chi_{n \alpha} i t+\nu_{\alpha} \cos \psi-i S_{n} \nu_{\alpha} \sin \psi\right)\left(\chi_{n \alpha} i t\right) \\
+ & i \frac{k_{\perp}^{2}}{k^{2}} \frac{\sin ^{2} \psi}{\left(1+i \zeta_{\alpha} t\right)}|n| \frac{T_{n}^{-}}{T_{n}^{+}} I_{|n|+1}\left(\frac{S_{n \alpha}(t)}{1+i \zeta_{\alpha} t}\right) \\
\times & \left(i \chi_{n \alpha} i t-\nu_{\alpha} \cos \psi-i S_{n} \nu_{\alpha} \sin \psi\right)\left(\chi_{n \alpha} i t\right) \\
= & i \frac{k_{\perp}^{2}}{k^{2}} \frac{\sin ^{2} \psi}{\left(1+i \zeta_{\alpha} t\right)}|n| I_{|n|+1}\left(\frac{S_{n \alpha}(t)}{1+i \zeta_{\alpha} t}\right)\left(\chi_{n \alpha} i t\right) \\
+\nu_{\alpha} \cos \psi & \left.\left(\frac{T_{n}^{+}}{T_{n}^{-}}-\frac{T_{n}^{-}}{T_{n}^{+}}\right)-i S_{n} \nu_{\alpha} \sin \psi\left(\frac{T_{n}^{+}}{T_{n}^{-}}+\frac{T_{n}^{-}}{T_{n}^{+}}\right)\right] \\
= & 2 \frac{k_{\perp}^{2}}{k^{2}} \frac{\sin ^{2} \psi}{\left(1+i \zeta_{\alpha} t\right)}|n| I_{|n|+1}\left(\frac{T_{n \alpha}}{T_{n}^{+}}\right) \\
& \times \frac{H_{n \alpha}(t)}{S_{n \alpha}(t)}\left[\chi_{n \alpha}(i t)+S_{n} \nu_{\alpha} \sin \psi\right] .
\end{aligned}
$$

The last two terms in $\Lambda^{\dagger}$ can be written as follows,

$$
\begin{gathered}
-\frac{k_{\|} k_{\perp}}{k^{2}} \frac{\sin \psi N_{\|}}{\left(1+i \zeta_{\alpha} t\right)} \mu_{\alpha}^{1 / 2} \chi_{n \alpha} H_{n \alpha}(t) \frac{T_{n}^{-}}{T_{n}^{+}} I_{|n|+1}\left(\frac{S_{n \alpha}(t)}{1+i \zeta_{\alpha} t}\right)(i t)^{2} \\
-\frac{k_{\|} k_{\perp}}{k^{2}} \frac{\sin \psi N_{\|}}{\left(1+i \zeta_{\alpha} t\right)} \mu_{\alpha}^{1 / 2} \chi_{n \alpha} H_{n \alpha}(t) \frac{T_{n}^{+}}{T_{n}^{-}} I_{|n|+1}\left(\frac{S_{n \alpha}(t)}{1+i \zeta_{\alpha} t}\right)(i t)^{2} \\
=-\frac{k_{\|} k_{\perp}}{k^{2}} \frac{\sin \psi N_{\|}}{\left(1+i \zeta_{\alpha} t\right)} \mu_{\alpha}^{1 / 2} \chi_{n \alpha} H_{n \alpha}(t) I_{|n|+1}\left(\frac{S_{n \alpha}(t)}{1+i \zeta_{\alpha} t}\right)(i t)^{2} \\
\times\left(\frac{T_{n}^{+}}{T_{n}^{-}}+\frac{T_{n}^{-}}{T_{n}^{+}}\right) \\
=-2 \frac{k_{\|} k_{\perp}}{k^{2}} \frac{\sin \psi N_{\|}}{\left(1+i \zeta_{\alpha} t\right)} \mu_{\alpha}^{1 / 2} \chi_{n \alpha} I_{|n|+1}\left(\frac{S_{n \alpha}(t)}{1+i \zeta_{\alpha} t}\right)(i t)^{2} \\
\times \frac{H_{n \alpha}(t)}{S_{n \alpha}(t)}\left[\nu_{\alpha}^{2}-\chi_{n \alpha}^{2}(i t)^{2}\right] .
\end{gathered}
$$

Using Eqs. (36), (37) and (38),

$$
\begin{gathered}
\Lambda^{\dagger}==2 \frac{\chi_{n \alpha} \sin \psi}{\left(1+i \zeta_{\alpha} t\right)} I_{|n|+1}\left(\frac{S_{n \alpha}(t)}{1+i \zeta_{\alpha} t}\right) \frac{H_{n \alpha}(t)}{S_{n \alpha}(t)}\left\{\frac{k_{\perp}^{2}}{k^{2}} n \nu_{\alpha}(i t)\right. \\
\left.+\frac{k_{\perp}^{2}}{k^{2}}|n| \sin \psi \chi_{n \alpha}(i t)^{2}-\frac{k_{\|} k_{\perp}}{k^{2}} N_{\|} \mu_{\alpha}^{1 / 2}(i t)^{2}\left[\nu_{\alpha}^{2}-\chi_{n \alpha}^{2}(i t)^{2}\right]\right\} .
\end{gathered}
$$


Using this expression in Eq. (28),

$$
\begin{aligned}
& \varepsilon_{l}=1-\sum_{\alpha} \frac{\omega_{\alpha}^{2}}{\omega^{2}} \mu_{\alpha} \sum_{n \rightarrow-\infty}^{\infty}\left\{\frac{k_{\perp}^{2}}{k^{2}} n^{2} \mathcal{G}_{0,1,|n|-1,|n|}\right. \\
& +\frac{k_{\|}^{2}}{k^{2}}\left(\mathcal{G}_{0,1,|n|,|n|}+\mu_{\alpha} N_{\|}^{2} \mathcal{G}_{2,1,|n|,|n|}\right) \\
& -2 \frac{k_{\perp}^{2}}{k^{2}} n \nu_{\alpha} \chi_{n \alpha} \sin \psi \mathcal{G}_{1,2,|n|-1,|n|} \\
& +2 \frac{k_{\perp}^{2}}{k^{2}} \chi_{n \alpha}^{2} \sin ^{2} \psi\left[\mathcal{G}_{2,3,|n|,|n|}+|n| \mathcal{G}_{2,2,|n|-1,|n|}\right. \\
& \left.-|n| \mathcal{G}_{2,2,|n|,|n|+1}-\nu_{\alpha}^{2} \mathcal{G}_{2,3,|n|,|n|+1}+\chi_{n \alpha}^{2} \mathcal{G}_{4,3,|n|,|n|+1}\right] \\
& -\frac{2 k_{\|} k_{\perp}}{k^{2}} N_{\|}|n| \mu_{\alpha}^{1 / 2}\left[S_{n} \nu_{\alpha} \mathcal{G}_{1,1,|n|-1,|n|}-\sin \psi \chi_{n \alpha} \mathcal{G}_{2,1,|n|-1,|n|}\right] \\
& \left.+2 \frac{k_{\|} k_{\perp}}{k^{2}} N_{\|} \chi_{n \alpha} \mu_{\alpha}^{1 / 2} \sin \psi \mathcal{G}_{2,2,|n|,|n|}\right\} \\
& +i \sum_{\alpha} \frac{\omega_{\alpha}^{2}}{\omega^{2}} \mu_{\alpha}^{2} \sum_{n \rightarrow-\infty}^{\infty} \int_{0}^{\infty} d t \mathrm{e}^{i \mu_{\alpha}\left(1-n Y_{\alpha}\right) t} e^{-\mu_{\alpha} N_{\|}^{2} t^{2} / 2} \\
& \times \frac{\mathrm{e}^{-\left(\nu_{\alpha}^{2}+\chi_{n \alpha}^{2} t^{2}\right) /\left(1+i \zeta_{\alpha} t\right)}}{\mu_{\alpha}\left(1+i \zeta_{\alpha} t\right)} \frac{\left[H_{n \alpha}(t)\right]^{(|n|-1)}}{\left(S_{n \alpha}(t)\right)^{|n|}} \\
& \times 2 \frac{\chi_{n \alpha} \sin \psi}{\left(1+i \zeta_{\alpha} t\right)} I_{|n|+1}\left(\frac{S_{n \alpha}(t)}{1+i \zeta_{\alpha} t}\right) \frac{H_{n \alpha}(t)}{S_{n \alpha}(t)}\left\{\frac{k_{\perp}^{2}}{k^{2}} n \nu_{\alpha}(i t)\right. \\
& \left.+\frac{k_{\perp}^{2}}{k^{2}}|n| \sin \psi \chi_{n \alpha}(i t)^{2}-\frac{k_{\|} k_{\perp}}{k^{2}} N_{\|} \mu_{\alpha}^{1 / 2}(i t)^{2}\left[\nu_{\alpha}^{2}-\chi_{n \alpha}^{2}(i t)^{2}\right]\right\} \text {. } \\
& \varepsilon_{l}=1-\sum_{\alpha} \frac{\omega_{\alpha}^{2}}{\omega^{2}} \mu_{\alpha} \sum_{n \rightarrow-\infty}^{\infty}\left\{\frac{k_{\perp}^{2}}{k^{2}} n^{2} \mathcal{G}_{0,1,|n|-1,|n|}\right. \\
& +\frac{k_{\|}^{2}}{k^{2}}\left(\mathcal{G}_{0,1,|n|,|n|}+\mu_{\alpha} N_{\|}^{2} \mathcal{G}_{2,1,|n|,|n|}\right) \\
& -2 \frac{k_{\perp}^{2}}{k^{2}} n \nu_{\alpha} \chi_{n \alpha} \sin \psi \mathcal{G}_{1,2,|n|-1,|n|} \\
& +2 \frac{k_{\perp}^{2}}{k^{2}} \chi_{n \alpha}^{2} \sin ^{2} \psi\left[\mathcal{G}_{2,3,|n|,|n|}+|n| \mathcal{G}_{2,2,|n|-1,|n|}\right. \\
& \left.-|n| \mathcal{G}_{2,2,|n|,|n|+1}-\nu_{\alpha}^{2} \mathcal{G}_{2,3,|n|,|n|+1}+\chi_{n \alpha}^{2} \mathcal{G}_{4,3,|n|,|n|+1}\right] \\
& -\frac{2 k_{\|} k_{\perp}}{k^{2}} N_{\|}|n| \mu_{\alpha}^{1 / 2}\left[S_{n} \nu_{\alpha} \mathcal{G}_{1,1,|n|-1,|n|}-\sin \psi \chi_{n \alpha} \mathcal{G}_{2,1,|n|-1,|n|}\right] \\
& +2 \frac{k_{\|} k_{\perp}}{k^{2}} N_{\|} \chi_{n \alpha} \mu_{\alpha}^{1 / 2} \sin \psi \mathcal{G}_{2,2,|n|,|n|} \\
& +2 \frac{k_{\perp}^{2}}{k^{2}} n \nu_{\alpha} \chi_{n \alpha} \sin \psi \mathcal{G}_{1,2,|n|,|n|+1} \\
& +2 \frac{k_{\perp}^{2}}{k^{2}}|n| \chi_{n \alpha}^{2} \sin ^{2} \psi \mathcal{G}_{2,2,|n|,|n|+1}-2 \frac{k_{\|} k_{\perp}}{k^{2}} N_{\|} \mu_{\alpha}^{1 / 2} \chi_{n \alpha} \sin \psi \\
& \left.\times\left[\nu_{\alpha}^{2} \mathcal{G}_{2,2,|n|,|n|+1}-\chi_{n \alpha}^{2} \mathcal{G}_{4,2,|n|,|n|+1}\right]\right\} . \\
& \varepsilon_{l}=1-\sum_{\alpha} \frac{\omega_{\alpha}^{2}}{\omega^{2}} \mu_{\alpha} \sum_{n \rightarrow-\infty}^{\infty}\left\{\frac{k_{\perp}^{2}}{k^{2}} n^{2} \mathcal{G}_{0,1,|n|-1,|n|}\right.
\end{aligned}
$$




$$
\begin{gathered}
+\frac{k_{\|}^{2}}{k^{2}}\left(\mathcal{G}_{0,1,|n|,|n|}+\mu_{\alpha} N_{\|}^{2} \mathcal{G}_{2,1,|n|,|n|}\right) \\
-2 \frac{k_{\perp}^{2}}{k^{2}} n \nu_{\alpha} \chi_{n \alpha} \sin \psi\left[\mathcal{G}_{1,2,|n|-1,|n|}-\mathcal{G}_{1,2,|n|,|n|+1]}\right] \\
+2 \frac{k_{\perp}^{2}}{k^{2}} \chi_{n \alpha}^{2} \sin ^{2} \psi\left[\mathcal{G}_{2,3,|n|,|n|}+|n| \mathcal{G}_{2,2,|n|-1,|n|}\right. \\
\left.-\nu_{\alpha}^{2} \mathcal{G}_{2,3,|n|,|n|+1}+\chi_{n \alpha}^{2} \mathcal{G}_{4,3,|n|,|n|+1}\right] \\
-\frac{2 k_{\|} k_{\perp}}{k^{2}} N_{\|} n \mu_{\alpha}^{1 / 2} \nu_{\alpha} \mathcal{G}_{1,1,|n|-1,|n|} \\
-2 \frac{k_{\|} k_{\perp}}{k^{2}} N_{\|} \mu_{\alpha}^{1 / 2} \chi_{n \alpha} \sin \psi\left[-\mathcal{G}_{2,2,|n|,|n|}-|n| \mathcal{G}_{2,1,|n|-1,|n|}\right. \\
\left.\left.+\nu_{\alpha}^{2} \mathcal{G}_{2,2,|n|,|n|+1}-\chi_{n \alpha}^{2} \mathcal{G}_{4,2,|n|,|n|+1}\right]\right\}
\end{gathered}
$$

An interesting limiting case to be considered is the case of propagation perpendicular to the direction of the magnetic field and of the inhomogeneity. In this case from Eq. (39) we obtain,

$$
\begin{gathered}
\varepsilon_{l}=1-\sum_{\alpha} \frac{\omega_{\alpha}^{2}}{\omega^{2}} \mu_{\alpha} \sum_{n \rightarrow-\infty}^{\infty}\left\{n^{2} \mathcal{G}_{0,1,|n|-1,|n|}\right. \\
-2 n \nu_{\alpha} \chi_{n \alpha}\left[\mathcal{G}_{1,2,|n|-1,|n|}-\mathcal{G}_{1,2,|n|,|n|+1}\right] \\
\quad+2 \chi_{n \alpha}^{2}\left[|n| \mathcal{G}_{2,2,|n|-1,|n|}+\mathcal{G}_{2,3,|n|,|n|}\right. \\
\left.\left.-\nu_{\alpha}^{2} \mathcal{G}_{2,3,|n|,|n|+1}+\chi_{n \alpha}^{2} \mathcal{G}_{4,3,|n|,|n|+1}\right]\right\}
\end{gathered}
$$

which, as expected, corresponds to the expression for the component $\varepsilon_{22}$ of the effective dielectric tensor, for a Maxwellian distribution function for particles of type $\alpha$, in the non-relativistic approximation [5].

\section{Acknowledgments}

The present research was supported by Brazilian agencies CNPq and FAPERGS. L.F.Z. acknowledges useful discussions with $\mathrm{M}$. Bornatici, and thanks the hospitality of Instituto di Fisica A. Volta, Universitá di Pavia, Italy, during part of the North Hemisphere Winter of 2003.

\section{References}

[1] L. F. Ziebell and R. S. Schneider, in X Latin American Workshop on Plasma Physics combined with VII Encontro Brasileiro de Física de Plasmas, SBF (SBF, São Pedro, SP, Brasil, 2003), pp.93-, oral presentation.

[2] L. F. Ziebell and R. S. Schneider, accepted for publication in the Braz. J. Phys., (2004).

[3] O. J. G. Silveira, L. F. Ziebell, R. S. Schneider, and R. Gaelzer, in in X Latin American Workshop on Plasma Physics combined with VII Encontro Brasileiro de Física de Plasmas, SBF (SBF, São Pedro, SP, Brasil, 2003), pp.43-, poster presentation.

[4] R. Gaelzer, R. S. Schneider, and L. F. Ziebell, Phys. Rev. E 51, 2407 (1995).

[5] R.Gaelzer, R. S. Schneider, and L. F. Ziebell, Phys. Rev. E 55, 5859 (1997).

[6] T. H. Stix, The theory of plasma waves (AIP, New York, 1992).

[7] V. S. Beskin, A. V. Gurevich, and Y. I. Istomin, Sov. Phys. JETP 65, 715 (1987).

[8] R. A. Caldela Filho, R. S. Schneider, and L. F. Ziebell, J. Plasma Phys. 42, 165 (1989).

[9] M. Abramowitz and I. A. Stegun, Handbook of Mathematical Functions (Dover Publications, New York, 1970). 